\title{
Functional renormalization with fermions and tetrads
}

\author{
P. Donà and R. Percacci \\ International School for Advanced Studies, \\ via Bonomea 265, 34136 Trieste, Italy and \\ INFN, Sezione di Trieste, Italy
}

\begin{abstract}
We investigate some aspects of the renormalization group flow of gravity in the presence of fermions, which have remained somewhat puzzling so far. The first is the sign of the fermionic contribution to the running of Newton's constant, which depends on details of the cutoff. We argue that only one of the previously used schemes correctly implements the cutoff on eigenvalues of the Dirac operator, and it acts in the sense of screening Newton's constant. We also show that Kähler fermions give the same contribution to the running of the cosmological and Newton constant as four Dirac spinors. We then calculate the graviton contributions to the beta functions by imposing the cutoffs on the irreducible spin components of the tetrad. In this way we can probe the gauge dependence of the off-shell flow. The results resemble closely those of the metric formalism, except for an increased scheme- and (off shell) gauge-dependence.
\end{abstract}

\section{CUTOFF SCHEMES}

The functional renormalization group equation (FRGE) is a convenient method to calculate the quantum effective action that is not restricted to renormalizable field theories [1, 2]. Instead of computing directly the effective action, one computes its derivative with respect to an external mass scale $k$ called "the cutoff". This "beta functional" is automatically free of UV and IR divergences. The advantage of this procedure is that it provides a definition of the beta functions of a theory that does not refer to any UV regularization. Then, taking some (generally local) action as starting point at an UV scale $\Lambda$, one can obtain the effective action by integrating the FRGE from $\Lambda$ to zero. The divergences of the theory reappear when one tries to shift $\Lambda$ to infinity and can be analysed by integrating the FRGE in the direction of increasing $k$.

For our purposes the most useful implementation of this idea is the Wetterich equation [2]. It gives the $k$-dependence of a functional called the Effective Average Action (EAA), which is a modified effective action where the propagation of the low momentum modes is suppressed. Here "low momentum" generally means "momenta below a given scale $k$ ". The suppression is described, in flat space, by some function $R_{k}\left(q^{2}\right)$, where $q^{2}$ is the squared momentum. To be admissible, the 
cutoff function $R_{k}$ must satisfy the following basic requirements: (i) it must be continuous and monotonically decreasing in $q^{2}$ and $k$, (ii) it must go rapidly to zero for $q^{2}>k^{2}$, (iii) it must tend to a positive (possibly infinite) value for $q^{2} \rightarrow 0$ and (iv) it must tend to zero for $k \rightarrow 0$. It is unavoidable that details of the flow depend on the shape of the cutoff function, even though the effective action, which is obtained by letting $k \rightarrow 0$, is not. This is referred to as "scheme dependence" and is akin to the renormalization scheme dependence in perturbation theory.

When one considers anything more than a single scalar field, in addition to the shape of the function $R_{k}(z)$, one encounters further choices. We will be concerned mainly with gravity, which offers a particularly complex scenario. The application of the FRGE to gravity has been pioneered in [3], followed by [4]. Already in these early works the implementation of the cutoff followed different methods. In [3] the cutoff was imposed on the tracefree and trace parts of the metric fluctuation (a decomposition that is purely algebraic), whereas in [4] the cutoff was imposed separately on each irreducible spin component (a finer decomposition that involves differential conditions). Following [5], we will call the former a cutoff of type "a" and the latter of type "b". When gravity is involved, the kinetic operators governing the propagation of quantum fields typically have the structure $-\nabla^{2}+a R+b E$, where $\nabla$ is the Riemannian covariant derivative, $R$ stands here for some combination of curvature terms (Riemann, Ricci and scalar curvatures, with indices arranged in various ways) and $E$ a term that may involve other background fields and possibly also couplings. Since Fourier analysis is not available in general curved spaces, in defining the cutoff one has to specify an operator whose eigenvalues form a basis in field space and in general play the role that plane waves play in flat space. One takes $R_{k}$ to be a function of this operator (or rather, its eigenvalues). In [5] the following terminology has been introduced: a cutoff is of type I if $R_{k}$ is a function of $-\nabla^{2}$, of type II if it is a function of $-\nabla^{2}+a R$ and of type III if it is a function of $-\nabla^{2}+a R+b E$. It is clear that there are infinitely many more possible cutoff variants and that this classification is very incomplete, but it covers the most commonly used cutoffs.

The RG flow in the so-called Einstein-Hilbert truncation, where one retains in the action terms at most linear in curvature, has been studied in [6-10], with en extended ghost sector in [11 13] and using the Vilkoviski-de Witt formalism in [14]. Higher derivative terms have been analysed in [5, 15 20]. The contribution of matter fields has been taken into account in [4, 21 24] using a Type I cutoff and in [25] using a type II cutoff. Conversely, the effect of gravity on fermionic interactions has been studied in [26 29].

Physical observables, which are related to the full effective action at $k=0$, will be independent of the cutoff choice. Furthermore, some terms in the beta functional, namely those that refer 
to dimensionless couplings, can actually be shown to be scheme-independent. It is also wellunderstood that if the system has a nontrivial fixed point, its position is not universal. In general, the scheme-dependence of the flow should therefore not be a cause of major concern [30].

In the literature on the renormalization group for gravity, there is however one result where the scheme dependence seems to be particularly nasty: it is the contribution of fermion fields to the beta function of Newton's constant. This contribution has been computed for example in section III of [5]. If there are $n_{D}$ Dirac fields it is

$$
-n_{D} \frac{1}{2} \frac{1}{(4 \pi)^{2}} \int d^{4} x \sqrt{g}\left(\frac{1}{6} Q_{1}\left(\frac{\partial_{t} R_{k}}{P_{k}}\right)-\frac{1}{4} Q_{2}\left(\frac{\partial_{t} R_{k}}{P_{k}^{2}}\right)\right) 4 R
$$

if one uses a type I cutoff and

$$
-n_{D} \frac{1}{2} \frac{1}{(4 \pi)^{2}} \int d^{4} x \sqrt{g}\left(\frac{1}{6}-\frac{1}{4}\right) Q_{1}\left(\frac{\partial_{t} R_{k}}{P_{k}}\right) 4 R=\frac{n_{D}}{96 \pi^{2}} Q_{1}\left(\frac{\partial_{t} R_{k}}{P_{k}}\right) \int d^{4} x \sqrt{g} R
$$

if one uses a type II cutoff. Here $Q_{n}(W)=\frac{1}{\Gamma(n)} \int_{0}^{\infty} d z z^{n-1} W(z)$. If we use the optimized cutoff [9]

$$
R_{k}(z)=\left(k^{2}-z\right) \theta\left(k^{2}-z\right)
$$

we get $Q_{1}\left(\frac{\partial_{t} R_{k}}{P_{k}}\right)=2 k^{2}, Q_{2}\left(\frac{\partial_{t} R_{k}}{P_{k}^{2}}\right)=k^{2}$ and therefore the contribution of fermions to the relevant term of the beta functional is

$$
\begin{array}{rr}
-\frac{n_{D}}{96 \pi^{2}} \int d^{4} x \sqrt{g} R & \text { for the type I cutoff } \\
\frac{n_{D}}{48 \pi^{2}} \int d^{4} x \sqrt{g} R & \text { for the type II cutoff. }
\end{array}
$$

The latter agrees with an earlier independent calculation of the renormalization of $G$ in [31]; the former differs not just in the value of the coefficient but even in the sign. ${ }^{1}$ To check that this is not just a quirk of the optimized cutoff, consider an exponential cutoff

$$
R_{k}(z)=\frac{z e^{-a\left(z / k^{2}\right)^{b}}}{1-e^{-a\left(z / k^{2}\right)^{b}}}
$$

In order to guarantee that condition (iii) is satisfied, one has to assume $b \geq 1$. For example if $b=1$, $Q_{1}\left(\frac{\partial_{t} R_{k}}{P_{k}}\right)=\pi^{2} k^{2} / 3 a$ and $Q_{2}\left(\frac{\partial_{t} R_{k}}{P_{k}^{2}}\right)=2 k^{2} / a$ and therefore we encounter the same problem, independently of the parameter $a$. One can see that the same is true also for $b>1$.

This sign ambiguity is puzzling. Since the beta function of $G$ vanishes for $G=0$, the sign of $G$ can never change in the course of the RG flow (we disregard here as pathological the case when

\footnotetext{
${ }^{1}$ This was noted a while ago by Calmet et al. while they were working on [32]. R.P. wishes to thank D. Reeb for correspondence on this point.
} 
the inverse of $G$ passes through zero). Therefore, if the physical RG trajectory approaches a fixed point in the UV, the sign of Newton's constant at low energy will be the same as that of Newton's constant at the fixed point. In a model where gravity is induced by minimally coupled fermions, the sign of Newton's constant would depend on the scheme.

One has to be careful in drawing physical conclusions from these calculations: the relation between the coupling $G$ appearing in $\Gamma_{k}$ and the physical Newton's constant that is measured in the lab may not be as simple as it seems. The functional that obeys an exact RG equation is not a simple functional of a single metric but rather a functional of a background metric and (the expectation value of) the fluctuation of the metric. This functional is not invariant under shifts of the background and fluctuation that keep the sum constant, and therefore there may be several couplings that one could legitimately call "Newton's constant" (for a discussion see [33]). The one that we are discussing here is the one that multiplies the Hilbert action constructed from the background metric only, and it is not obvious that the other ones would behave in the same way. In order to make a completely well-defined statement one should really calculate a physical observable and in such a calculation all ambiguities should disappear. It is therefore possible, in principle, that even the sign difference between the two calculations discussed above may be resolved when one considers physical observables. In this paper we will argue for a different and simpler solution of the issue, namely that only the type II cutoff gives a result with the physically correct sign.

As an aside, we will also calculate the contribution to the beta functions of the cosmological constant and Newton constant due to Euclidean Kähler fermions in four dimensions. Kähler fermions are a way of describing fermion fields in terms of Grassmann algebra elements, instead of spinors and has the merit that it does not require the use of tetrad fields. We find that one Kähler fermion gives exactly the same contribution as four Dirac spinors, and that the same sign issue is present.

When gravity is coupled to fermions an additional question arises regarding the field carrying the gravitational degrees of freedom. In [4, 21, 22], where the contribution of graviton loops was added to that of matter fields, as well as in [26 28] where scalars and fermions were interacting with gravity, the carrier of the gravitational degrees of freedom was the metric. It has been pointed out in [34] that in the presence of fermions it may be more natural to use the tetrad. Even if one chooses a Lorentz gauge where the antisymmetric part of the tetrad fluctuation is suppressed, the two calculations are not the same, because one has to work off shell and the Hessian in the tetrad formalism contains some additional terms proportional to the equations of motion. The calculations in [34], which used a type Ia cutoff in the $\alpha=1$ gauge, show that a fixed point is still present, but is less stable than in the metric formulation. We will compute the gravitational contributions in 
the tetrad formalism, using type b cutoffs (both Ib and IIb); this allows us to analyse the off-shell $\alpha$-dependence. The results will be found to be somewhat closer to those of the metric formalism than those found in [34] (which we have independently verified), but they still present a stronger dependence on the scheme and on the gauge parameter.

\section{KÄHLER FERMIONS}

Before discussing in detail the issue of cutoff scheme with ordinary spinor fermions, we would like to point out that precisely the same problem arises also in another representation of fermionic matter. This section is not strictly necessary for the following discussion so readers that are mainly interested in the solution of the puzzle presented above may go directly to section III.

As is well-known, in any dimension the Grassmann and the Clifford algebras are isomorphic as vector spaces. This is the basis for a representation of fermion fields as inhomogeneous differential forms [35-37]. Such fields are called Kähler fermions. In this representation the analogue of the Dirac operator is the first order operator $d+\delta$, where $d$ is the exterior derivative and $\delta$ is its adjoint. Note that the definition does not require the use of a tetrad. We would like to compare the contribution of Kähler fermions to the gravitational beta functions to the one of ordinary spinor fermions. In particular we would like to see whether the same sign issue arises when different cutoff types are used. Since the details of the calculation are strongly dimension-dependent, we shall restrict our attention to the case $d=4$.

An inhomogeneous complex differential form $\Phi$ can be expanded as

$\Phi=\varphi(x)+\varphi_{\mu}(x) d x^{\mu}+\frac{1}{2 !} \varphi_{\mu \nu}(x) d x^{\mu} \wedge d x^{\nu}+\frac{1}{3 !} \varphi_{\mu \nu \rho}(x) d x^{\mu} \wedge d x^{\nu} \wedge d x^{\rho}+\frac{1}{3 !} \varphi_{\mu \nu \rho \sigma}(x) d x^{\mu} \wedge d x^{\nu} \wedge d x^{\rho} \wedge d x^{\sigma}$

We can map the 3- and 4-form via Hodge duality into a 1- and 0-form respectively. The field $\Phi$ thus describes a scalar, a pseudoscalar, a vector, a pseudovector and an antisymmetric tensor, for a total of 16 complex components. This is an early sign of the fact that one Kähler field is equivalent to four Dirac fields.

The square of the Kähler operator is the Laplacian on forms: $\Delta=(d+\delta)^{2}=d \delta+\delta d$. On forms of degree 0,1 and 2 it is given explicitly by

$$
\begin{aligned}
\Delta^{(0)} & =-\nabla^{2} \\
\Delta_{\nu}^{(1) \mu} & =-\nabla^{2} \delta_{\nu}^{\mu}+R^{\mu}{ }_{\nu} \\
\Delta_{\alpha \beta}^{(2) \gamma \sigma} & =-\nabla^{2} \mathbf{1}_{\alpha \beta}{ }^{\gamma \sigma}+R_{\alpha}{ }^{\gamma} \delta_{\beta}{ }^{\sigma}-R_{\beta}{ }^{\gamma} \delta_{\alpha}{ }^{\sigma}-2 R_{\alpha}{ }^{\gamma}{ }^{\sigma}{ }^{\sigma}
\end{aligned}
$$


In order to read off the beta functions of the cosmological constant and Newton's constant it enough to consider a spherical (Euclidean de Sitter) background, with curvature tensor

$$
R_{\mu \nu \rho \sigma}=\frac{1}{d(d-1)}\left(g_{\mu \rho} g_{\nu \sigma}-g_{\mu \sigma} g_{\nu \rho}\right) R ; \quad R_{\mu \nu}=\frac{1}{d} g_{\mu \nu} R
$$

Then the operators defined above reduce to

$$
\begin{aligned}
\Delta_{\nu}^{(1) \mu} & =\left(-\nabla^{2}+\frac{1}{4} R\right) \delta_{\nu}^{\mu} \\
\Delta_{\alpha \beta}^{(2) \gamma \sigma} & =\left(-\nabla^{2}+\frac{1}{3} R\right) \mathbf{1}_{\alpha \beta}{ }^{\gamma \sigma}
\end{aligned}
$$

The ERGE for a Kähler fermion with a type II cutoff is

$$
\begin{aligned}
\frac{d \Gamma_{k}}{d t} & =-2 \frac{1}{2} \operatorname{Tr}_{(0)}\left(\frac{\partial_{t} R_{k}\left(\Delta^{(0)}\right)}{P_{k}\left(\Delta^{(0)}\right)}\right)-2 \frac{1}{2} \operatorname{Tr}_{(1)}\left(\frac{\partial_{t} R_{k}\left(\Delta^{(1)}\right)}{P_{k}\left(\Delta^{(1)}\right)}\right)-\frac{1}{2} \operatorname{Tr}_{(2)}\left(\frac{\partial_{t} R_{k}\left(\Delta^{(2)}\right)}{P_{k}\left(\Delta^{(2)}\right)}\right) \\
& =4 \cdot \frac{1}{2} \frac{1}{(4 \pi)^{2}} \int d^{4} x \sqrt{g}\left[-4 Q_{2}\left(\frac{\partial_{t} R_{k}}{P_{k}}\right)+\frac{1}{3} R Q_{1}\left(\frac{\partial_{t} R_{k}}{P_{k}}\right)\right] .
\end{aligned}
$$

For a type I cutoff we find instead:

$$
\begin{aligned}
\frac{d \Gamma_{k}}{d t} & =-2 \cdot \frac{1}{2} \operatorname{Tr}_{(0)}\left(\frac{\partial_{t} R_{k}\left(-\nabla^{2}\right)}{P_{k}\left(-\nabla^{2}\right)}\right)-2 \cdot \frac{1}{2} \operatorname{Tr}_{(1)}\left(\frac{\partial_{t} R_{k}\left(-\nabla^{2}\right)}{P_{k}\left(-\nabla^{2}\right)+\frac{R}{4}}\right)-\frac{1}{2} \operatorname{Tr}_{(2)}\left(\frac{\partial_{t} R_{k}\left(-\nabla^{2}\right)}{P_{k}\left(-\nabla^{2}\right)+\frac{R}{3}}\right) \\
& =4 \cdot \frac{1}{2} \frac{1}{(4 \pi)^{2}} \int d^{4} x \sqrt{g}\left[-4 Q_{2}\left(\frac{\partial_{t} R_{k}}{P_{k}}\right)-R\left(\frac{2}{3} Q_{1}\left(\frac{\partial_{t} R_{k}}{P_{k}}\right)-Q_{2}\left(\frac{\partial_{t} R_{k}}{P_{k}^{2}}\right)\right)\right] .
\end{aligned}
$$

Evaluating the $Q$-functionals for the cutoff (3) we get:

$$
\begin{aligned}
& \text { Type II: } \quad \frac{d \Gamma_{k}}{d t}=4 \cdot \frac{1}{2} \frac{1}{(4 \pi)^{2}} \int d^{4} x \sqrt{g}\left[-4 k^{4}+\frac{2}{3} R k^{2}\right] \\
& \text { Type I: } \quad \frac{d \Gamma_{k}}{d t}=4 \cdot \frac{1}{2} \frac{1}{(4 \pi)^{2}} \int d^{4} x \sqrt{g}\left[-4 k^{4}-\frac{1}{3} R k^{2}\right]
\end{aligned}
$$

In both cases the effect of one Kähler fermion is seen to match exactly the result of four spinors $\left(n_{D}=4\right)$. This should not induce one to believe that spinors and Kähler fermions are equivalent: in fact, their contributions to the curvature squared terms are quite different. Nevertheless, the puzzling sign issue of the $R$-term that afflicts spinor fermions is present in this case too.

\section{CUTOFF CHOICE FOR FERMIONS}

We now return to ordinary Dirac spinor fields and we reexamine in more detail their contribution to the gravitational effective action and beta functions. For the sake of generality we will now work in arbitrary dimension $d$. The standard way of defining the effective action for a fermion field that is minimally coupled to gravity is to exploit the properties of the logarithm and write

$$
\Gamma=-\operatorname{Tr} \log (|\not D|)=-\frac{1}{2} \operatorname{Tr} \log \left(\not D^{2}\right)=-\frac{1}{2} \operatorname{Tr} \log \left(-\nabla^{2}+\frac{R}{4}\right) .
$$


The corresponding EAA can then be defined as

$$
\Gamma_{k}=-\frac{1}{2} \operatorname{Tr} \log \left(-\nabla^{2}+\frac{R}{4}+R_{k}\right) .
$$

In the definition of this functional one encounters the same ambiguities that we have mentioned earlier for bosonic systems. In addition to the shape of the cutoff function $R_{k}$, one seems to also have the freedom of choosing the argument of this function to be either $-\nabla^{2}$ (type I cutoff) or $-\nabla^{2}+\frac{R}{4}$ (type II cutoff). The former choice has been made in [4, 21 24], the latter in [25]. Taking the $t$-derivative (where $\left.t=\log \left(k / k_{0}\right)\right)$ and defining $P_{k}(z)=z+R_{k}(z)$, one has

$$
\begin{array}{cc}
\frac{d \Gamma_{k}}{d t}=-\frac{1}{2} \operatorname{Tr} \frac{\partial_{t} R_{k}\left(-\nabla^{2}\right)}{P_{k}\left(-\nabla^{2}\right)+\frac{R}{4}} & \text { for a type I cutoff } \\
\frac{d \Gamma_{k}}{d t}=-\frac{1}{2} \operatorname{Tr} \frac{\partial_{t} R_{k}\left(-\nabla^{2}+\frac{R}{4}\right)}{P_{k}\left(-\nabla^{2}+\frac{R}{4}\right)} & \text { for a type II cutoff. }
\end{array}
$$

The first few terms in the curvature expansion of these traces can be evaluated, for any background, using heat kernel methods. However, for a spherical background, the spectrum of the Dirac operator is known explicitly and the same traces can also be computed directly as spectral sums. Comparison of these calculations indicates that only the type II cutoff correctly reflects the cutoff on eigenvalues of the Dirac operator.

\section{A. Heat kernel evaluation}

With a type I cutoff, the trace (19) giving contribution of $n_{D}$ Dirac spinors to the FRGE is

$$
\frac{d \Gamma_{k}}{d t}=-\frac{n_{D}}{2} \frac{2^{[d / 2]}}{(4 \pi)^{d / 2}} \int d^{d} x \sqrt{g}\left[Q_{\frac{d}{2}}\left(\frac{\partial_{t} R_{k}}{P_{k}}\right)+\left(\frac{1}{6} Q_{\frac{d}{2}-1}\left(\frac{\partial_{t} R_{k}}{P_{k}}\right)-\frac{1}{4} Q_{\frac{d}{2}}\left(\frac{\partial_{t} R_{k}}{P_{k}^{2}}\right)\right) R+\ldots\right] .
$$

Here $2^{[d / 2]}$ (where $[x]$ is the integer part of $x$ ) is the dimension of the representation. The first term proportional to $R$ is proportional to the heat kernel coefficient $b_{2}\left(-\nabla^{2}\right)$, and the second comes from the expansion of the denominator in (19). Evaluating the $Q$-functionals with the cutoff (3) we obtain

$$
\frac{d \Gamma_{k}}{d t}=-\frac{1}{\Gamma\left(\frac{d}{2}+1\right)} \frac{2^{[d / 2]}}{(4 \pi)^{d / 2}} n_{D} \int d^{d} x \sqrt{g}\left[k^{d}+\frac{d-3}{12} k^{d-2} R\right] .
$$

Evaluating (20) with the same techniques yields

$$
\frac{d \Gamma_{k}}{d t}=-\frac{n_{D}}{2} \frac{2^{[d / 2]}}{(4 \pi)^{d / 2}} \int d^{d} x \sqrt{g}\left[Q_{\frac{d}{2}}\left(\frac{\partial_{t} R_{k}}{P_{k}}\right)-\frac{1}{12} R Q_{\frac{d}{2}-1}\left(\frac{\partial_{t} R_{k}}{P_{k}}\right)+\ldots\right],
$$


where the term proportional to $R$ comes entirely from the heat kernel coefficient $b_{2}\left(-\nabla^{2}+\frac{R}{4}\right)$. Evaluating with the cutoff (3i) we obtain

$$
\frac{d \Gamma_{k}}{d t}=-\frac{1}{\Gamma\left(\frac{d}{2}+1\right)} \frac{2^{[d / 2]}}{(4 \pi)^{d / 2}} n_{D} \int d^{d} x \sqrt{g}\left[k^{d}-\frac{d}{24} k^{d-2} R\right] .
$$

In $d=4$ this yields the results quoted in section I. We see that the sign issue is present in any dimension $d>3$.

\section{B. Spectral sums on $S^{d}$}

The heat kernel calculation of the preceding subsection can be done in an arbitrary background. On the other hand, in the case of the spherical background we know explicitly the spectrum of the Dirac operator: the eigenvalues and multiplicities are

$$
\lambda_{n}^{ \pm}= \pm \sqrt{\frac{R}{d(d-1)}}\left(\frac{d}{2}+n\right), \quad m_{n}=2^{\left[\frac{d}{2}\right]}\left(\begin{array}{c}
n+d-1 \\
n
\end{array}\right), \quad n=0,1, \ldots
$$

With this information one can compute the trace of any function of the Dirac operator as $\operatorname{Tr} f(\not D)=$ $\sum_{n=0}^{\infty} m_{n} f\left(\lambda_{n}\right)$. We will now evaluate the r.h.s. of the FRGE by imposing a cutoff on the eigenvalues of the Dirac operator. The EAA can be defined directly in terms of the Dirac operator as

$$
\Gamma_{k}=-\operatorname{tr} \log \left(|\not D|+R_{k}^{D}(|\not D|)\right)
$$

where the cutoff $R_{k}^{D}$ has to be a function of the modulus of the Dirac operator, since we want to suppress the modes depending on the wavelength of the corresponding eigenfunctions. This is also needed for reasons of convergence. Since the operator is first order, the conditions that $R_{k}^{D}$ has to satisfy are similar to (i)-(iv) of section I, except for the replacement of $q^{2}$ and $k^{2}$ by $\lambda_{n}$ and $k$ respectively. For the explicit evaluation, we will use the optimized profile

$$
R_{k}^{D}(z)=(k-z) \theta(k-z), \quad(z>0)
$$

Then we have

$$
\operatorname{Tr}\left[\frac{\partial_{t} R_{k}^{D}(|\not D|)}{P_{k}^{D}(|\not D|)}\right]=\sum_{n} m_{n} \frac{\partial_{t} R_{k}^{D}\left(\left|\lambda_{n}\right|\right)}{P_{k}^{D}\left(\left|\lambda_{n}\right|\right)}=\sum_{ \pm} \sum_{n} m_{n} \theta\left(k-\left|\lambda_{n}\right|\right) .
$$

The sum can be computed using the Euler-Maclaurin formula. Details are given in Appendix I. The result is

$$
\frac{d \Gamma_{k}}{d t}=-\operatorname{Tr}\left[\frac{\partial_{t} R_{k}^{D}}{P_{k}^{D}}\right]=-\frac{1}{\Gamma\left(\frac{d}{2}+1\right)} \frac{2^{\left[\frac{d}{2}\right]}}{(4 \pi)^{\frac{d}{2}}} V(d)\left(k^{d}-\frac{d}{24} k^{d-2} R+O\left(R^{2}\right)\right),
$$


where $V(d)$ is the volume of the $d$-sphere. This agrees exactly with (24), which was obtained with the type II cutoff. (We have checked that the agreement extends also to the next order in the curvature expansion.)

\section{Discussion}

Note that computing the r.h.s. of the FRGE with a spectral sum is a much more direct procedure, since it avoids going through the square root of the square of the Dirac operator, and also avoids having to use the Laplace transform and the heat kernel. It is therefore also a more reliable procedure when there are ambiguities. The agreement of the spectral sum with the type II-heat kernel calculation is a useful consistency check and suggests that the latter gives the correct result whereas the type I cutoff does not.

If so, there remain to understand why the type I cutoff should not be admissible in this case. One can get some hint by thinking of what this cutoff does in terms of eigenvalues of the Dirac operator. We begin by noting that (26) can be rewritten as follows: ${ }^{2}$

$$
\Gamma_{k}=-\frac{1}{2} \operatorname{tr} \log \left(|\not D|+R_{k}^{D}(|\not D|)\right)^{2}=-\frac{1}{2} \operatorname{tr} \log \left(-\nabla^{2}+\frac{R}{4}+2|\not D| R_{k}^{D}(|\not D|)+R_{k}^{D}(|\not D|)^{2}\right) .
$$

One can compare this with (18). Note that the cutoff $R_{k}$ in that formula could be a function of different operators which, on a sphere, differ by a constant shift. For the present purposes it is convenient to think of it as a function of $\not D^{2}$. Calling $\bar{R}_{k}$ this function and calling $z=|\not D|$, we have

$$
\bar{R}_{k}\left(z^{2}\right)=2 z R_{k}^{D}(z)+R_{k}^{D}(z)^{2}
$$

We can solve this relation to get

$$
R_{k}^{D}(z)=-z+\sqrt{z^{2}+\bar{R}_{k}\left(z^{2}\right)}
$$

so for any cutoff imposed at the level of (18) one can reverse-engineer an effective cutoff to be imposed at the level of (26) that will give the same result.

In general, this cutoff may fail to satisfy the required conditions, in particular condition (iv). For a type II cutoff, $R_{k}$ in (18) is a function of $z^{2}$, so $\bar{R}_{k}\left(z^{2}\right)=R_{k}\left(z^{2}\right)$. This implies that $\bar{R}_{0}\left(z^{2}\right)=0$ and thus also $R_{0}^{D}(z)=0$ for all $z>0$. For a type I cutoff, on the other hand, this may not be the case, as we will show in the following examples.

\footnotetext{
${ }^{2}$ This is a formal relation because the functional $\Gamma_{k}$ is ill-defined, but one can write a corresponding relation for $\partial_{t} \Gamma_{k}$, with the same result.
} 
Consider first the optimized cutoffs. In the type II case one has $\bar{R}_{k}\left(z^{2}\right)=\left(k^{2}-z^{2}\right) \theta\left(k^{2}-z^{2}\right)$ and one finds that in this case the corresponding cutoff $R_{k}^{D}(z)$ given by (32) is also optimized, and precisely of the form (27). This is a way of understanding why the two calculations give the same result. In the case of a type I cutoff, we have instead $\bar{R}_{k}\left(z^{2}\right)=\left(k^{2}-z^{2}+R / 4\right) \theta\left(k^{2}-z^{2}+R / 4\right)$, whence one derives

$$
R_{k}^{D}(z)=\left(\sqrt{k^{2}+\frac{R}{4}}-z\right) \theta\left(\sqrt{k^{2}+\frac{R}{4}}-z\right) .
$$

This does not tend uniformly to zero when $k \rightarrow 0$. In the case of an exponential type II cutoff with $\bar{R}_{k}=R_{k}$ given by (6), we have

$$
R_{k}^{D}(z)=-z+\frac{z}{\sqrt{1-e^{-a\left(z^{2} / k^{2}\right)^{b}}}}
$$

which has all the desired properties. On the other hand for an exponential type I cutoff with $\bar{R}_{k}$ given by (6),

$$
R_{k}^{D}(z)=-z+\sqrt{z^{2}+\left(z^{2}-\frac{R}{4}\right) \frac{e^{-a\left(z^{2}-\frac{R}{4}\right)^{b} / k^{2 b}}}{1-e^{-a\left(z^{2}-\frac{R}{4}\right)^{b} / k^{2 b}}}}
$$

For $b$ odd, and in particular for the most natural case $b=1$, this function does not tend uniformly to zero when $k \rightarrow 0$ and therefore condition (iv) is not satisfied. ${ }^{3}$

These arguments lend support to the view that only the type II cutoff gives the physically correct result. Of course not all results obtained from the type I cutoff have to be wrong, for example the leading term (renormalizing the cosmological constant) and, in $d=4$, the curvature squared terms, are the same using the two cutoffs. These however are just the "universal" quantities that do not depend on the choice of the cutoff. We believe that for the generic dimensionful, non-universal quantities, the results obtained via a type I cutoff should not be trusted.

\section{TETRAD GRAVITY}

We will now compute the graviton contribution to the running of Newton's constant and cosmological constant, in $d$-dimensions, when the tetrad is used as a field variable. This has been discussed recently in [34] using a type Ia cutoff, i.e. a cutoff that depends on $-\nabla^{2}$ that is added to the full gravitational Hessian. In order to have a manageable, minimal Laplacian-type operator,

\footnotetext{
${ }^{3}$ Note that in the limit $k \rightarrow 0$ the function $R_{k}^{D}$ is non zero only for $z<\sqrt{R / 4}$. Since the smallest eigenvalue of the Dirac operator is $\sqrt{R / 3}$, it remains true that $\lim _{k \rightarrow 0} \Gamma_{k}=\Gamma$.
} 
this requires that the gravitational gauge-fixing parameter be fixed to $\alpha=1$. We will use instead cutoffs of type $b$, meaning that the graviton is first decomposed into irreducible components of spin 2, 1 and 0 , and the cutoff is imposed separately one each component. This permits the discussion of general diffeomorphism gauges. We will use both type Ib and type IIb cutoffs.

\section{A. Hessian and gauge fixing}

The ansatz we make for the effective average action is the standard Einstein-Hilbert truncation

$$
\Gamma_{k}[e, \bar{e}]=\Gamma_{k}^{E H}[e, \bar{e}]+\Gamma_{k}^{G F}[e, \bar{e}]=-\frac{1}{16 \pi G_{k}} \int d^{d} x \operatorname{det} e\left(R(g(e))-2 \Lambda_{k}\right)+\Gamma_{k}^{G F}[e, \bar{e}]
$$

where we have indicated the $k$-dependence of the couplings and $\Gamma_{k}^{G F}$ is the gauge-fixing term, to be specified below. In tetrad formulation the metric is represented in terms of vielbeins $e_{\mu}^{a}$ as $g_{\mu \nu}=e^{a}{ }_{\mu} e^{b}{ }_{\nu} \eta_{a b}$. If we decompose $g_{\mu \nu} \equiv \bar{g}_{\mu \nu}+h_{\mu \nu}$ and $e^{a}{ }_{\mu} \equiv \bar{e}_{\mu}^{a}+\varepsilon^{a}{ }_{\mu}$ we have the relation

$$
h_{\mu \nu}=2 \varepsilon_{(\mu \nu)}+\varepsilon_{(\mu}^{\rho} \varepsilon_{\nu) \rho}
$$

where Latin indices on $\varepsilon$ have been transformed to Greek ones by contraction with $\bar{e}$. Now substituting this formula in the Taylor expansion of the metric in terms of metric fluctuations we find:

$$
\Gamma^{E H}(e)=\Gamma^{E H}(\bar{e})+\int \frac{\delta \Gamma^{E H}}{\delta g_{\mu \nu}} 2 \varepsilon_{\mu \nu}+\zeta \int \frac{\delta \Gamma^{E H}}{\delta g_{\mu \nu}} \varepsilon_{\mu}{ }^{\rho} \varepsilon_{\nu \rho}+\frac{1}{2} \iint \frac{\delta \Gamma^{E H}}{\delta g_{\mu \nu} \delta g_{\rho \sigma}} 4 \varepsilon_{\mu \nu} \varepsilon_{\rho \sigma}+\ldots
$$

In the third term we have introduced by hand a factor $0 \leq \zeta \leq 1$ that interpolates continuously between the pure metric formalism $\left(\zeta=0, h_{\mu \nu}=2 \varepsilon_{(\mu \nu)}\right)$ and the tetrad formalism $(\zeta=1)$. We see that the part of the action quadratic in $\varepsilon$ differs from the one in the metric formalism by terms proportional to the equations of motion. Since in the derivation of the beta functions it is essential to work off shell, we cannot ignore these terms.

The gauge fixing terms for diffeomorphisms and local Lorentz transformations are

$$
\Gamma_{k}^{G F}[e, \bar{e}]=\frac{1}{2 \alpha} \int \mathrm{d}^{\mathrm{d}} x \sqrt{\bar{g}} \bar{g}^{\mu \nu} F_{\mu} F_{\nu}+\frac{1}{2 \alpha_{L}} \int \mathrm{d}^{\mathrm{d}} x \sqrt{\bar{g}} G^{a b} G_{a b}
$$

For diffeomorphisms we choose the condition

$$
F_{\mu}=\frac{1}{\sqrt{16 \pi G}}\left(\bar{\nabla}^{\nu} \bar{h}_{\mu \nu}-\frac{\beta}{2} \bar{\nabla}_{\mu} \bar{h}\right)
$$

while for the internal $O(d)$ transformation we choose a symmetric vielbein $G^{a b}=\varepsilon^{[a b]}$. We will choose $\alpha_{L}=0$ in order to simplify the computation. This correspond to a sharp implementation of 
the Lorentz gauge fixing, where one can simply set $\varepsilon_{[\mu \nu]}=0$ and suppress the corresponding rows and columns in the Hessian.

Next we perform the TT decomposition on the symmetric part of the vielbein fluctuation

$$
\varepsilon_{(\mu \nu)}=h_{\mu \nu}^{T T}+\nabla_{\mu} \xi_{\nu}+\nabla_{\nu} \xi_{\mu}+\nabla_{\mu} \nabla_{\nu} \sigma-\frac{1}{d} g_{\mu \nu} \nabla^{2} \sigma+\frac{1}{d} g_{\mu \nu} h^{2}
$$

and the associated field redefinitions $\xi_{\mu} \rightarrow \sqrt{-\nabla^{2}-\frac{R}{d}} \xi_{\mu}$ and $\sigma \rightarrow \sqrt{\left(-\nabla^{2}-\frac{R}{(d-1)}\right)\left(-\nabla^{2}\right)} \sigma$.

With these definitions, and dropping bars from the background quantities for notational simplicity, the quadratic part of the action (36) is

$$
\begin{aligned}
\Gamma_{h^{T} h^{T}}^{(2)}= & \frac{1}{2} \int \sqrt{g} h^{T \mu \nu}\left[-\nabla^{2}+\left(\frac{d(d-3)+4}{d(d-1)}-\zeta \frac{d-2}{2 d}\right) R-(2-\zeta) \Lambda\right] h_{\mu \nu}^{T} \\
\Gamma_{\xi \xi}^{(2)}= & \frac{1}{\alpha} \int \sqrt{g} \xi^{\nu}\left[-\nabla^{2}+\left(\frac{\alpha(d-2)-1}{d}-\zeta \alpha \frac{d-2}{2 d}\right) R-\alpha(2-\zeta) \Lambda\right] \xi_{\nu} \\
\Gamma_{\sigma \sigma}^{(2)}= & \frac{(d-1)}{2 d} \frac{2(d-1)-\alpha(d-2)}{\alpha d} \times \\
& \int \sqrt{g} \sigma\left[-\nabla^{2}+\frac{(d-2)(2-\zeta) \alpha-4}{2(d-1)-\alpha(d-2)} R-\frac{\alpha d(2-\zeta)}{2(d-1)-\alpha(d-2)} \Lambda\right] \sigma \\
\Gamma_{h h}^{(2)}= & \frac{d-2}{4 d} \frac{2\left(d^{2}-3 d+2\right) \alpha-(d \beta-2)^{2}}{d \alpha(d-2)} \times \\
& \int \sqrt{g} h\left[-\nabla^{2}+\frac{\alpha(d-2)(d-4+\zeta)}{2\left(d^{2}-3 d+2\right) \alpha-(d \beta-2)^{2}} R-2 \frac{d \alpha(d-2+\zeta)}{2\left(d^{2}-3 d+2\right) \alpha-(d \beta-2)^{2}} \Lambda\right] h \\
\Gamma_{h \sigma}^{(2)}= & \frac{(d-2) \alpha+d \beta-2}{d \alpha} \frac{(d-1)}{d} \int \sqrt{g} h\left(-\nabla^{2}-\frac{R}{(d-1)}\right) \nabla^{2} \sigma
\end{aligned}
$$

We notice that for $\beta=\frac{1}{d}(\alpha(d-2)+2)$ we can get rid of the mixed term. In the rest of the paper we will work in this "diagonal" gauge. In this case the trace part reduces to

$$
\Gamma_{h h}^{(2)}=-\frac{1}{2} \frac{d-2}{2 d} \int \sqrt{g} h\left[-\nabla^{2}+\frac{d-4+\zeta}{2(d-1)-\alpha(d-2)} R-\frac{2 d}{2(d-1)-\alpha(d-2)}\left(1+\frac{\zeta}{d-2}\right) \Lambda\right] h
$$

After decomposing the diffeomorphism ghost in its transverse and longitudinal parts, and absorbing $\sqrt{-\nabla^{2}}$ in the latter, the ghost action is the sum of

$$
\Gamma_{\bar{c}_{\nu}^{T} c_{\mu}^{T}}^{(2)}=\int \sqrt{g} \bar{c}_{\nu}^{T}\left(\nabla^{2}+\frac{R}{d}\right) c_{\mu}^{T} \quad \Gamma_{\bar{c} c}^{(2)}=\int \sqrt{g} \bar{c}\left(\nabla^{2}+2 \frac{R}{d}\right) c
$$

The Lorentz ghosts do not propagate and following standard perturbative procedure one could neglect them entirely, but we will follow [34] and introduce a cutoff for them too. The corresponding contribution to the FRGE is computed, together with other traces, in Appendix II.

\section{B. Beta functions}

The FRGE can now be calculated by introducing a cutoff separately in each spin sector. (This is known as a "type b" cutoff.) Using the same heat kernel methods that we have employed in section 
IIA the expression for $\partial_{t} \Gamma_{k}$ can be expanded to linear order in $R$. Comparing the terms of order zero and one in $R$ in the FRGE yields:

$$
\begin{aligned}
\partial_{t}\left(\frac{2 \Lambda}{16 \pi G}\right) & =\frac{k^{d}}{16 \pi}\left(A_{1}+\eta A_{2}\right) \\
-\partial_{t}\left(\frac{1}{16 \pi G}\right) & =\frac{k^{d-2}}{16 \pi}\left(B_{1}+\eta B_{2}\right)
\end{aligned}
$$

where $\eta=-\partial_{t} G / G$ and $A_{i}, B_{i}$ are in general polynomials in $\tilde{\Lambda}=k^{-2} \Lambda$. From here one can find the beta functions of the dimensionless parameters $\tilde{G}=k^{d-2} G$ and $\tilde{\Lambda}$ :

$$
\begin{aligned}
& \partial_{t} \tilde{G}=(d-2) \tilde{G}+\frac{B_{1} \tilde{G}^{2}}{1+\tilde{G} B_{2}} \\
& \partial_{t} \tilde{\Lambda}=-2 \tilde{\Lambda}+\tilde{G} \frac{A_{1}+2 B_{1} \tilde{\Lambda}+\tilde{G}\left(A_{1} B_{2}-A_{2} B_{1}\right)}{2\left(1+B_{2} \tilde{G}\right)}
\end{aligned}
$$

In the following two sections we will give explicit results using specific cutoffs.

For numerical results in $d=4$ we will always use the optimized cutoff (3). For a discussion of the dependence on the shape of the function $R_{k}(z)$ we refer to [34]. We will instead concentrate on the differences between cutoffs of type I vs. II and type a vs b. For the type Ia case we refer again to the extensive discussion in [34], whose results we have checked independently. We will report in detail the results for the cases Ib and IIb, and highlight the differences with the case Ia.

\section{Type Ib cutoff}

First we choose as reference operator, in each spin sector, the "bare" Laplacian $-\nabla^{2}$. The cutoff is a function $R_{k}\left(-\nabla^{2}\right)$. This is called a cutoff of type Ib. The calculation of the coefficients $A_{1}$, $A_{2}, B_{1}$ and $B_{2}$ for arbitrary dimension and cutoff shape is described in Appendix A. Here we just report the result in $d=4$ and for the cutoff profile (3):

$$
\begin{aligned}
A_{1}=\frac{1}{2 \pi} & {\left[\frac{5}{1-(2-\zeta) \tilde{\Lambda}}+\frac{3}{1-\alpha(2-\zeta) \tilde{\Lambda}}+\frac{1}{1-\frac{2 \alpha(2-\zeta)}{3-\alpha} \tilde{\Lambda}}+\frac{1}{1-\frac{2(2+\zeta)}{3-\alpha} \tilde{\Lambda}}-8\right]+A^{L}(\tilde{\mu}) } \\
A_{2}=\frac{1}{12 \pi} & {\left[\frac{5}{1-(2-\zeta) \tilde{\Lambda}}+\frac{3}{1-\alpha(2-\zeta) \tilde{\Lambda}}+\frac{1}{1-\frac{2 \alpha(2-\zeta)}{3-\alpha} \tilde{\Lambda}}+\frac{1}{1-\frac{2(2+\zeta)}{3-\alpha} \tilde{\Lambda}}\right] } \\
B_{1}=\frac{1}{24 \pi} & {\left[-\frac{20}{1-(2-\zeta) \tilde{\Lambda}}-\frac{5(8-3 \zeta)}{(1-(2-\zeta) \tilde{\Lambda})^{2}}+\frac{6}{1-\alpha(2-\zeta) \tilde{\Lambda}}+\frac{9(1-\alpha(2-\zeta))}{4(1-\alpha(2-\zeta) \tilde{\Lambda})^{2}}\right.} \\
& +\frac{4}{1-\frac{2 \alpha(2-\zeta) \tilde{\Lambda}}{3-\alpha}}+\frac{12+6 \alpha(\zeta-2)}{(3-\alpha)\left(1-\frac{2 \alpha(2-\zeta) \tilde{\Lambda}}{3-\alpha}\right)^{2}}+\frac{4}{1-\frac{2(\zeta+2) \tilde{\Lambda}}{3-\alpha}}-\frac{6 \zeta}{(3-\alpha)\left(1-\frac{2(\zeta+2) \tilde{\Lambda}}{3-\alpha}\right)^{2}} \\
& -50]+B^{L}(\tilde{\mu})
\end{aligned}
$$




$$
\begin{aligned}
B_{2}=\frac{1}{144 \pi}[ & -\frac{30}{1-(2-\zeta) \tilde{\Lambda}}-\frac{5(8-3 \zeta)}{(1-(2-\zeta) \tilde{\Lambda})^{2}}+\frac{9}{1-\alpha(2-\zeta) \tilde{\Lambda}}+\frac{9(1-\alpha(2-\zeta)}{(1-\alpha(2-\zeta) \tilde{\Lambda})^{2}} \\
& \left.+\frac{6}{1-\frac{2 \alpha(2-\zeta) \tilde{\Lambda}}{3-\alpha}}+\frac{12+6 \alpha(\zeta-2)}{(3-\alpha)\left(1-\frac{2 \alpha(2-\zeta) \tilde{\Lambda}}{3-\alpha}\right)^{2}}+\frac{6}{1-\frac{2(\zeta+2) \tilde{\Lambda}}{3-\alpha}}-\frac{6 \zeta}{(3-\alpha)\left(1-\frac{2(\zeta+2) \tilde{\Lambda}}{3-\alpha}\right)^{2}}\right] .
\end{aligned}
$$

The result is still quite general: it depends on the parameter $\zeta$, which allows us to interpolate continuously between the purely metric formulation $(\zeta=0)$ and the purely tetrad formulation $(\zeta=1)$, on the arbitrary gauge parameter $\alpha$, which allows us to test the gauge dependence of the results, and on the parameter $\mu$ that allows us to weigh differently the contribution of the Lorentz ghosts.

Let us now describe the main features of these flows. Both in the metric and in the tetrad formulations, a UV-attractive fixed point is found for all values of $\mu$ and for $\alpha$ not too large. Its location and the corresponding critical exponents $\vartheta$ (which are defined as minus the eigenvalues of the stability matrix) are given in table I in the metric $(\zeta=0)$ or tetrad $(\zeta=1)$ formalism, in the gauges $\alpha=0, \alpha=1$ and with two different values of the dimensionless Lorentz ghost parameter $\tilde{\mu}=\mu / k: \tilde{\mu}=\infty$, and $\tilde{\mu}=1.2$. The former corresponds to neglecting the Lorentz ghosts entirely and the latter is chosen to ease comparison with [34], who found that this value gives results that are closest to the metric formalism in the gauge and scheme they use. Note that the case $\zeta=0$, $\alpha=1$ correspond to the purely metric flow with type Ib cutoff, which had already been discussed previously in the literature. Indeed the second row in table I agrees with the third row in table II in [5].

Whereas with a type Ia cutoff the fixed point becomes UV-repulsive, and a limit cycle develops, for $\tilde{\mu}$ sufficiently large, with the type Ib cutoff it remains UV-attractive for arbitrarily large $\tilde{\mu}$. This is a nice feature of this scheme, because it means that the fixed point can also be found if one adopts the perturbative prescription of neglecting the Lorentz ghosts entirely. However, the results in the tetrad formalism match most closely those of the metric formalism when $\tilde{\mu}$ is chosen to be a bit larger than one. As with the type Ia cutoff, for $\tilde{\mu}$ smaller than a critical value $\tilde{\mu}_{c}$, the critical exponents become real. We find $\tilde{\mu}_{c} \approx 0.705$ for $\alpha=0$ and $\tilde{\mu}_{c} \approx 0.715$ for $\alpha=1$.

The dependence of the universal quantities on the gauge parameters is illustrated in Fig. 1. The slow decrease of the real part of the critical exponent for $0<\alpha<2$ is in agreement with earlier calculations in the metric formalism (see e.g. fig. 9 in [7]). The results of different schemes seem to converge for $\alpha \rightarrow 0$ which, we recall, is believed to give the physically most reliable picture. On the other hand when $\alpha$ is greater than some value of order 2 the fixed point becomes repulsive, reproducing the behavior that had been observed in [34] for large $\tilde{\mu}$. It is tempting to conjecture 


\begin{tabular}{l|l|l|l|l} 
Scheme & $\tilde{\Lambda}_{*}$ & $\tilde{G}_{*}$ & $\tilde{\Lambda}_{*} \tilde{G}_{*}$ & $\vartheta$ \\
\hline $\mathrm{Ib}, \zeta=0, \alpha=0$ & 0.1569 & 0.9028 & 0.1416 & $2.147 \pm 2.620 i$ \\
$\mathrm{Ib}, \zeta=0, \alpha=1$ & 0.1715 & 0.7012 & 0.1203 & $1.689 \pm 2.486 i$ \\
$\mathrm{Ib}, \zeta=1, \tilde{\mu}=\infty, \alpha=0$ & 0.2288 & 1.363 & 0.3119 & $2.086 \pm 2.042 i$ \\
$\mathrm{Ib}, \zeta=1, \tilde{\mu}=\infty, \alpha=1$ & 0.2478 & 0.9472 & 0.2347 & $0.595 \pm 3.753 i$ \\
$\mathrm{Ib}, \zeta=1, \tilde{\mu}=1.2, \alpha=0$ & 0.0691 & 1.518 & 0.1050 & $2.237 \pm 1.248 i$ \\
$\mathrm{Ib}, \zeta=1, \tilde{\mu}=1.2, \alpha=1$ & 0.0798 & 1.3196 & 0.1053 & $1.892 \pm 1.093 i$
\end{tabular}

TABLE I: The non trivial fixed point in the type Ib cutoff in metric $(\zeta=0)$ and tetrad $(\zeta=1)$ formalism, in the gauges $\alpha=0$ and $\alpha=1$ and with different weights of the Lorentz ghosts. Recall $\operatorname{Re}(\vartheta)>0$ implies that the fixed point is UV attractive.

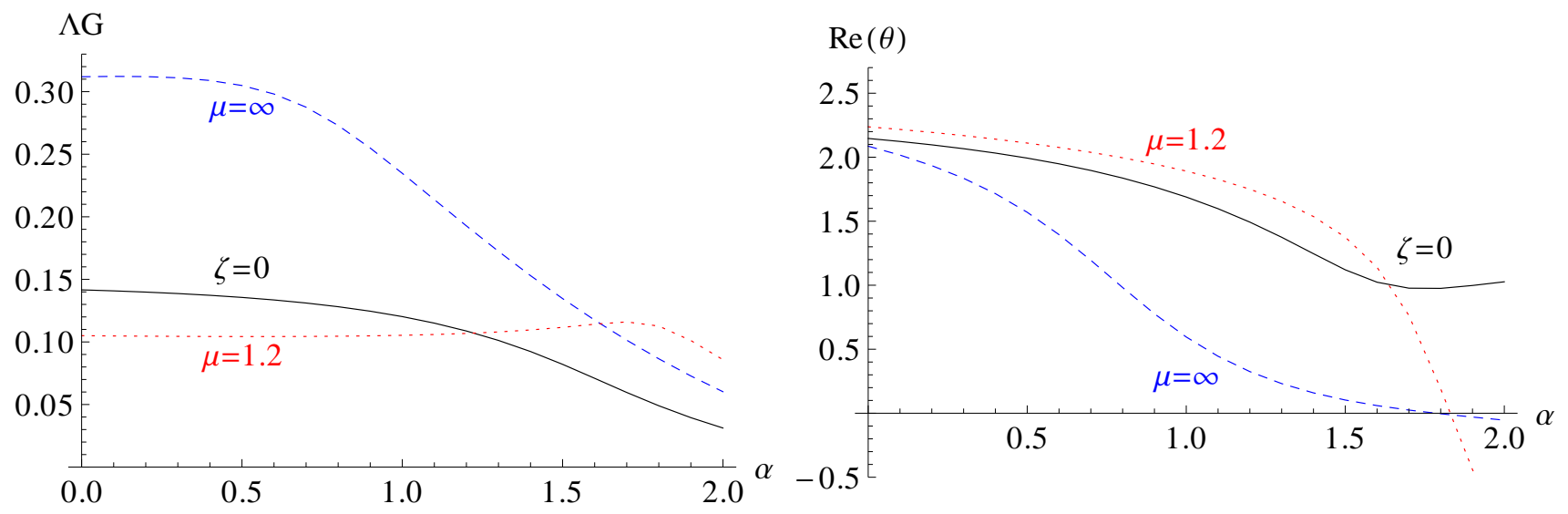

FIG. 1: Plot of universal quantities as functions of the gauge parameter $\alpha$ for type Ib cutoff. In the left panel the product $\Lambda_{*} G_{*}$, in the right panel the real part of the critical exponent.

that also in the cutoff scheme Ia used in [34] the fixed point would have the usual properties, even for large $\tilde{\mu}$, if one could choose $\alpha$ closer to zero. The strong $\tilde{\mu}$-dependence that had been observed there may be due to a particularly strong $\alpha$-dependence. Altogether it appears that with a type Ib cutoff, the tetrad formalism leads to results that are qualitatively similar to those of the metric formalism, and that the correspondence is best when $0<\alpha<1$ and the Lorentz ghosts are turned on, with a parameter $\tilde{\mu}$ that is a little larger than one.

\section{Type IIb cutoff}

We call type IIb a cutoff imposed separately on each spin-component of the graviton and taking as reference operator the Laplace-type operator that appears in the corresponding Hessian, including the curvature terms, but not the term proportional to the cosmological constant. The rationale 


\begin{tabular}{l|l|l|l|l} 
Scheme & $\tilde{\Lambda}_{*}$ & $\tilde{G}_{*}$ & $\tilde{\Lambda}_{*} \tilde{G}_{*}$ & $\vartheta$ \\
\hline $\mathrm{IIb}, \zeta=0, \alpha=0$ & 0.1052 & 0.7216 & 0.0759 & $2.562 \pm 1.566 i$ \\
$\mathrm{IIb}, \zeta=0, \alpha=1$ & 0.0924 & 0.5557 & 0.0513 & $2.424 \pm 1.270 i$ \\
$\mathrm{IIb}, \zeta=1, \tilde{\mu}=\infty, \alpha=0$ & 0.1406 & 1.0176 & 0.1431 & $2.595 \pm 1.131 i$ \\
$\mathrm{IIb}, \zeta=1, \tilde{\mu}=\infty, \alpha=1$ & 0.1369 & 0.8427 & 0.1154 & $2.300 \pm 0.991 i$ \\
$\mathrm{IIb}, \zeta=1, \tilde{\mu}=1.2, \alpha=0$ & 0.0394 & 1.0008 & 0.0398 & $2.640 \pm 0.730 i$ \\
$\mathrm{IIb}, \zeta=1, \tilde{\mu}=1.2, \alpha=1$ & 0.0361 & 0.8299 & 0.0300 & $2.547 \pm 0.634 i$
\end{tabular}

TABLE II: The non trivial fixed point in the type IIb cutoff in metric $(\zeta=0)$ and tetrad $(\zeta=1)$ formalism, in the gauges $\alpha=0$ and $\alpha=1$ and with different weights of the Lorentz ghosts.

for excluding the cosmological constant term is that the cosmological constant is a running coupling and if one included it in the reference operator, it would not remain fixed in the course of the flow. Here we choose a reference operator that remains fixed along the flow. ${ }^{4}$ As we have already seen in the case of the fermions, the type II cutoffs tend to give somewhat simpler final formulae than the corresponding type I cutoffs, because to leading order one always finds traces of the function $\partial_{t} R_{k} / P_{k}$ and it is not necessary to expand the denominators.

The coefficients $A_{1}, A_{2}$ are the same as with a type Ib cutoff and are given in (52)531). The coefficients $B_{1}$ and $B_{2}$ for arbitrary dimension and cutoff shape are given in Appendix III. In $d=4$ and for the cutoff profile (3), they become

$$
\begin{aligned}
& B_{1}=\frac{1}{12 \pi}\left[-\frac{10(10-3 \zeta)}{1-(2-\zeta) \tilde{\Lambda}}+\frac{6(4-3 \alpha(2-\zeta))}{1-\alpha(2-\zeta) \tilde{\Lambda}}+\frac{2-\frac{6 \zeta}{3-\alpha}}{1-2 \frac{2+\zeta}{3-\alpha} \tilde{\Lambda}}+\frac{14-6 \frac{4-\alpha \zeta}{3-\alpha}}{1-2 \alpha \frac{2-\zeta}{3-\alpha} \tilde{\Lambda}}-40\right]+B^{L} \\
& B_{2}=\frac{1}{48 \pi}\left[-\frac{5(10-3 \zeta)}{1-(2-\zeta) \tilde{\Lambda}}-\frac{3(4-3 \alpha(2-\zeta)}{1-\alpha(2-\zeta) \tilde{\Lambda}}-\frac{\left.2-\frac{6 \zeta}{3-\alpha}\right)}{1-2 \frac{2+\zeta}{3-\alpha} \tilde{\Lambda}}-\frac{14-6 \frac{4-\alpha \zeta}{3-\alpha}}{1-2 \alpha \frac{2-\zeta}{3-\alpha} \tilde{\Lambda}}\right]
\end{aligned}
$$

Table II gives the UV-attractive fixed point and the corresponding critical exponents in the metric $(\zeta=0)$ or tetrad $(\zeta=1)$ formalism, in the gauges $\alpha=0,1$, and with two different values of the Lorentz ghost parameter, $\tilde{\mu}=\infty$, and $\tilde{\mu}=1.2$.

The results are qualitatively similar to the ones obtained with the type Ib cutoff. This is in line with all the results obtained previously in the Einstein-Hilbert truncation. The non trivial FP exists and has complex critical exponents for all values of $\tilde{\mu}$ greater than a critical value $\tilde{\mu}_{c}$, which is approximately equal to 0.766 for $\alpha=0$ and 0.748 for $\alpha=1$. For small $\tilde{\mu}$ the FP moves towards negative values of $\tilde{\Lambda}$. For large $\tilde{\mu}$ the fixed point remains UV attractive, in contrast to the result

\footnotetext{
${ }^{4}$ Cutoffs that depend on the full Hessian, including the terms proportional to the cosmological constant, were called of type III in [5], where they have been applied to the Einstein-Hilbert truncation.
} 


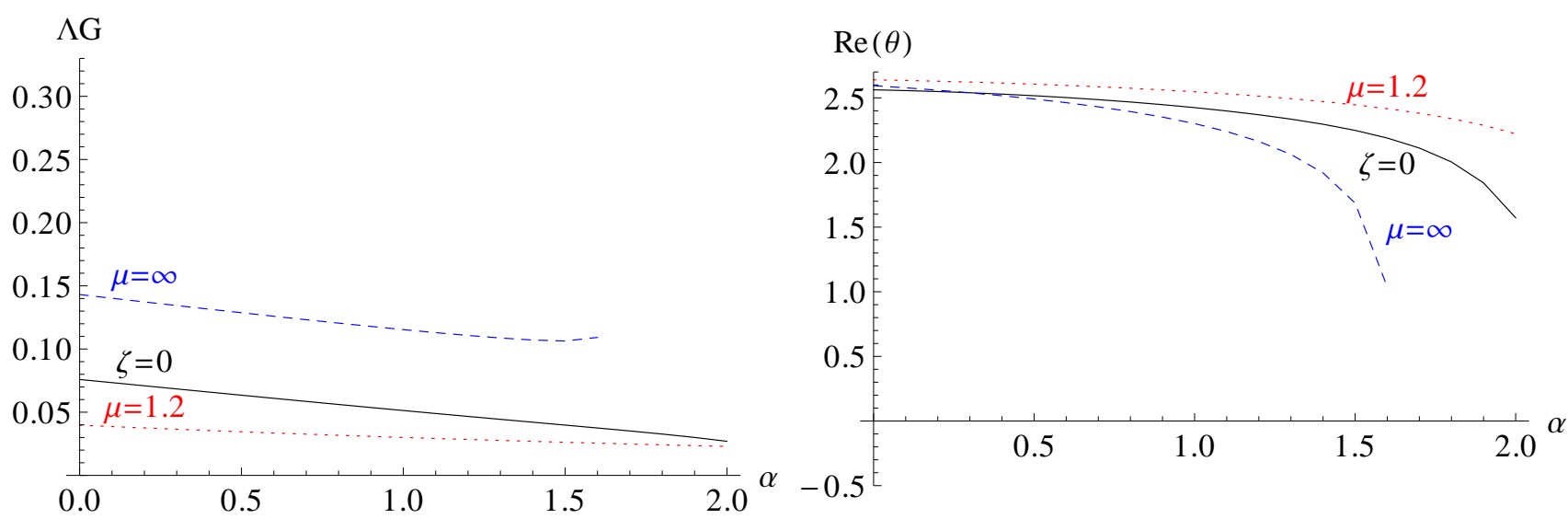

FIG. 2: Plot of universal quantities as functions of the gauge parameter $\alpha$ for type IIb cutoff. In the left panel the product $\Lambda_{*} G_{*}$, in the right panel the real part of the critical exponent.

found in [34] with the type Ia cutoff scheme (which we have independently verified). In particular we find that the FP has properties close to the standard ones of the metric formulation also when the Lorentz ghosts are neglected. Figure 2 gives the gauge-dependence of the universal quantities $\Lambda G$ and $\vartheta$. Note that the real part of the scaling exponent $\vartheta$ is particularly stable in this scheme, for $0<\alpha<1$.

\section{E. Type IIa cutoff}

For completeness we mention here also the results for the cutoff of type IIa, which had been discussed first in section IVC of [5]. In this scheme only the gauge $\alpha=1$ is easily computable. In this gauge it is enough to split the metric fluctuation into its trace and tracefree parts to write the Hessian of the Einstein-Hilbert action as two minimal Laplace-type operators. The cutoff is then defined as a function of these operators, including the curvature terms but excluding the cosmological constant term. This prescription leads to particularly simple expressions.

The coefficients $A_{1}, A_{2}$ are the same as with a other cutoff types considered here and are given in (5253). The coefficients $B_{1}$ and $B_{2}$ in $d=4$ and for the cutoff profile (3) are simply

$$
\begin{aligned}
& B_{1}=\frac{1}{12 \pi}\left[\frac{2-3 \zeta}{1-(2+\zeta) \tilde{\Lambda}}-\frac{27(2-\zeta)}{1-(2-\zeta) \tilde{\Lambda}}-40\right]+B^{L} \\
& B_{2}=\frac{1}{48 \pi}\left[\frac{2-3 \zeta}{1-(2+\zeta) \tilde{\Lambda}}-\frac{27(2-\zeta)}{1-(2-\zeta) \tilde{\Lambda}}\right]
\end{aligned}
$$

These expressions coincide with (56]57) when one puts $\alpha=1$ there. As a consequence, all properties of the flow are the same and we will not discuss this case further.

It is nevertheless interesting to understand the reason for this coincidence, which is not restricted 
to $d=4$ and is also independent of the shape of the function $R_{k} .{ }^{5}$ For the sake of simplicity we shall discuss here only the case $\zeta=0$, but the result is general. Since in all cases the trace field $h$ is treated separately, and its contribution is the same for type a and b cutoffs, it is enough to consider the tracefree part of the graviton, namely the components $h_{\mu \nu}^{T T}, \xi_{\mu}$ and $\sigma$. In the gauge $\alpha=1$, the Hessian in the tracefree subsector is a minimal second order operator of the form

$$
-\nabla^{2}+C_{T} R-2 \Lambda
$$

where $C_{T}=\frac{d(d-3)+4}{d(d-1)}$. When one uses a cutoff of type IIa (no further decomposition) the contribution of this sector to the r.h.s. of the FRGE is

$$
\sum_{n} \frac{\partial_{t} R\left(\lambda_{n}\right)-\eta R_{k}\left(\lambda_{n}\right)}{P_{k}\left(\lambda_{n}\right)-2 \Lambda}
$$

where $\lambda_{n}$ are the eigenvalues of the operator $\mathcal{O}=-\nabla^{2}+C_{T} R$. One can divide these eigenvalues into three classes, depending on the spin of the corresponding eigenfunction. Upon using the TT decomposition (41) one finds that the eigenvalues of $\mathcal{O}$ on fields of type $h_{\mu \nu}^{T T}, \nabla_{\mu} \xi_{\nu}-\nabla_{\nu} \xi_{\mu}$ and $\nabla_{\mu} \nabla_{\nu} \sigma-\frac{1}{d} \nabla^{2} \sigma$ are equal to the eigenvalues of the operators in square brackets in (42)4344), stripped of the $\Lambda$ terms. We denote these operators $\mathcal{O}^{T T}=-\nabla^{2}+C_{T} R, \mathcal{O}^{\xi}=-\nabla^{2}+C_{\xi} R$ and $\mathcal{O}^{\sigma}=-\nabla^{2}+C_{\sigma} R$ and the corresponding eigenvalues $\lambda_{n}^{T T}, \lambda_{n}^{\xi}$ and $\lambda_{n}^{\sigma}$. So, the trace (63) is equal to

$$
\sum_{n} \frac{\partial_{t} R\left(\lambda_{n}^{T T}\right)-\eta R_{k}\left(\lambda_{n}^{T T}\right)}{P_{k}\left(\lambda_{n}^{T T}\right)-2 \Lambda}+\sum_{n} \frac{\partial_{t} R\left(\lambda_{n}^{\xi}\right)-\eta R_{k}\left(\lambda_{n}^{\xi}\right)}{P_{k}\left(\lambda_{n}^{\xi}\right)-2 \Lambda}+\sum_{n} \frac{\partial_{t} R\left(\lambda_{n}^{\sigma}\right)-\eta R_{k}\left(\lambda_{n}^{\sigma}\right)}{P_{k}\left(\lambda_{n}^{\sigma}\right)-2 \Lambda}
$$

Since for $\alpha=1$ the coefficients of $\Lambda$ in (42/43) 44) are all the same and equal to -2 , this is recognized as the contribution of the fields $h_{\mu \nu}^{T T}, \xi_{\mu}$ and $\sigma$ to the r.h.s. of the FRGE when one uses a cutoff of type IIb. By a similar reasoning one also concludes that the ghost contribution is the same in the IIa and IIb schemes.

Things do not work in the same way for type I cutoffs, i.e. when the cutoff is a function of $-\nabla^{2}$. For a type Ia cutoff the contribution of the tracefree sector to the r.h.s. of the FRGE is

$$
\sum_{n} \frac{\partial_{t} R\left(\lambda_{n}\right)-\eta R_{k}\left(\lambda_{n}\right)}{P_{k}\left(\lambda_{n}\right)+C_{T} R-2 \Lambda}
$$

where $\lambda_{n}$ now denote the eigenvalues of $-\nabla^{2}$. This can be expanded as

$$
\sum_{n}^{T T} \frac{\partial_{t} R\left(\lambda_{n}\right)-\eta R_{k}\left(\lambda_{n}\right)}{P_{k}\left(\lambda_{n}\right)+C_{T} R-2 \Lambda}+\sum_{n}^{\xi} \frac{\partial_{t} R\left(\lambda_{n}\right)-\eta R_{k}\left(\lambda_{n}\right)}{P_{k}\left(\lambda_{n}\right)+C_{T} R-2 \Lambda}+\sum_{n}^{\sigma} \frac{\partial_{t} R\left(\lambda_{n}\right)-\eta R_{k}\left(\lambda_{n}\right)}{P_{k}\left(\lambda_{n}\right)+C_{T} R-2 \Lambda},
$$

\footnotetext{
${ }^{5}$ The agreement between cutoffs IIa and IIb for $\alpha=1$ had been noticed before in 38].
} 
where $\sum^{T T}, \sum^{\xi}$ and $\sum^{\sigma}$ denote the sum over eigenvalues of $-\nabla^{2}$ on $h_{\mu \nu}^{T T}, \nabla_{\mu} \xi_{\nu}-\nabla_{\nu} \xi_{\mu}$ and $\nabla_{\mu} \nabla_{\nu} \sigma-\frac{1}{d} \nabla^{2} \sigma$ respectively. On the other hand for a type Ib cutoff the same contribution is

$$
\sum_{n}^{T T} \frac{\partial_{t} R\left(\lambda_{n}\right)-\eta R_{k}\left(\lambda_{n}\right)}{P_{k}\left(\lambda_{n}\right)+C_{T} R-2 \Lambda}+\sum_{n}^{\xi} \frac{\partial_{t} R\left(\lambda_{n}\right)-\eta R_{k}\left(\lambda_{n}\right)}{P_{k}\left(\lambda_{n}\right)+C_{\xi} R-2 \Lambda}+\sum_{n} \frac{\partial_{t} R\left(\lambda_{n}\right)-\eta R_{k}\left(\lambda_{n}\right)}{P_{k}\left(\lambda_{n}\right)+C_{\sigma} R-2 \Lambda}
$$

One clearly sees that the two traces are different.

\section{DISCUSSION}

The implementation of the FRGE in the presence of fermions and gravity presents some subtleties that had not been fully appreciated until recently. The sign ambiguity of the fermionic contribution to the running of Newton's constant had been known for a while, but it was regarded as just another aspect of the scheme dependence that is intrinsic to applications of the FRGE, albeit a particularly worrying one. Although a completely satisfactory understanding can only come from a treatment of physical observables, we have argued here that the correct treatment of fermion fields, when the Dirac operator is squared, is to use a cutoff that depends on $-\nabla^{2}+\frac{R}{4}$ (type II cutoff). There also follows from our discussion that using a cutoff that depends on $-\nabla^{2}$ (type I cutoff) may give physically incorrect results. Unfortunately, several earlier studies (in particular [21, 22]) have used this scheme, so some of those results may have to be revised. We plan to return to this point in a future publication.

Another issue is the use of tetrad vs. metric degrees of freedom. We have extended the analysis of tetrad gravity initiated in [34] by using a different cutoff (type Ib and IIb vs. type Ia) which allowed us to keep the diffeomorphism gauge parameter $\alpha$ arbitrary. We have found that the results for the running couplings using the tetrad formalism are qualitatively similar to those of the metric formalism, with some quirks. The following points should be noted.

(i) For $\zeta=0$ one recovers the standard metric formalism. In the case of the type Ib cutoff the results agree with the ones obtained earlier in the literature [4, 5, 7]. The type IIb cutoff with generic $\alpha$ had never been used before and the results obtained here are new. We have shown that for $\alpha=1$ they coincide with the ones found in [5] for the type IIa cutoff. For other values of $\alpha$ they differ only marginally from those obtained with other cutoff types and confirm the stability of the fixed point in the metric formalism. Type II cutoffs have the attractive feature that they lead to somewhat more compact expressions for the beta functions.

ii) The case $\zeta=1$ corresponds to the tetrad formalism. In this case a new ambiguity appears in the definition of the ghost sector: it can be parameterized by a mass $\mu$ that appears in the mixing 
between diffeomorphism and Lorentz ghosts, or by the corresponding dimensionless parameter $\tilde{\mu}=\mu / k$. This parameter is a priori arbitrary, but in order not to introduce additional mass scales into the problem it is natural to assume that it is of order one. On the other hand we recall that in perturbation theory and in the chosen gauge the Lorentz ghosts are neglected, since they do not propagate. This corresponds to taking $\tilde{\mu}=\infty$.

iii) If one uses a type Ia cutoff and completely neglects the Lorentz ghosts, there is no attractive FP for positive $G$ [34]. Instead, one finds a UV-repulsive fixed point surrounded by a UV-attractive limit cycle ${ }^{6}$. This is not the case when one imposes the cutoff separately on each spin component, as we did here. We find that both with type Ib and IIb cutoffs an attractive FP with complex critical exponents is present also when Lorentz ghosts are neglected, both for $\alpha=1$ and $\alpha=0$. This is reassuring because in the metric formalism the fixed point can be found even using the perturbative one loop beta functions.

iv) If the contribution of Lorentz ghosts is added, as advocated in [34], its effect is weighted by the parameter $\tilde{\mu}$ : it is strong for small $\tilde{\mu}$ and weak for large $\tilde{\mu}$. Since the ghosts are fermions, the fixed point is shifted towards negative $\Lambda$ for decreasing $\tilde{\mu}$. In addition, they have a systematic effect on the critical exponents: the modulus of the imaginary part decreases with decreasing $\tilde{\mu}$ and there is a critical value $\tilde{\mu}_{c}$ under which the critical exponents become real. In the gauge $\alpha=1, \tilde{\mu}_{c}=0.715$ for a type Ib cutoff, $\tilde{\mu}_{c}=0.748$ for a type IIb cutoff, and $\tilde{\mu}_{c} \approx 0.8$ for a type Ia cutoff. Similar behaviour is observed also for $\alpha=0$. On the other hand, the real part of the critical exponent decreases with increasing $\tilde{\mu}$. With the type Ia cutoff this effect is most dramatic: the real part becomes negative for $\tilde{\mu} \approx 1.4$, and this marks the appearance of the limit cycle. With the type II cutoffs discussed here the effect is much weaker and the fixed point becomes only slightly less attractive even in the limit $\tilde{\mu} \rightarrow \infty$, both for $\alpha=1$ and $\alpha=0$.

v) The closest match between the tetrad and metric results is typically obtained if one chooses some value of $\tilde{\mu}$ that is not too far from one. This is always the case for the product $\Lambda G$, and in most cases also for the critical exponent. For the Ia cutoff this value was found to be approximately 1.2. For the Ib and IIb cutoffs it is somewhat larger, depending on the quantities one is comparing. An exception occurs for the critical exponent in the case of a cutoff of type IIb in the gauge $\alpha=0$, for which the best match occurs for $\tilde{\mu} \rightarrow \infty$.

vi) Using type b cutoffs (i.e. decomposing the fields into irreducible components) has the advantage that one can keep the diffeomorphism gauge parameter $\alpha$ arbitrary. The gauge dependence of the

\footnotetext{
${ }^{6}$ This is different from the behaviour of the limit cycle discussed in [39].
} 
critical exponents is similar to what had been observed previously in the metric formalism, as long as $\alpha$ is not too much greater than one. In particular the real part of the critical exponent tends to decrease as $\alpha$ increases, for small values of $\alpha$. In the limit $\alpha \rightarrow 0$ the $\tilde{\mu}$-dependence becomes very weak and the critical exponents nicely converges towards a common value. On the other hand for $\alpha$ somewhat larger than one the fixed point either becomes complex or repulsive. This is the behaviour that had been observed in [34] with the type Ia cutoff. This suggests that if we were able to compute the beta functions for this cutoff type with $\alpha \neq 1$ we would find that also with large $\tilde{\mu}$ the fixed point is present and has the standard properties for $\alpha$ sufficiently close to zero.

vii) Altogether the results are very similar to those found in the metric formalism, except for the dependence on the new parameter $\tilde{\mu}$, which is particularly strong for the type Ia cutoff. As argued in [34], one can probably attribute the increased sensitivity of the results to the fact that in the tetrad formalism one has to deal with more unphysical degrees of freedom. The type Ia cutoff seems to be particularly sensitive to the off-shell, unphysical features of the flow. Type b cutoffs, where each spin component is treated separately, are less sensitive.

viii) All of the preceding discussion is in the context of a "single metric truncation", i.e. one assumes that the VEV of the fluctuation field is zero. As discussed in [33], the application of the FRGE to gravity requires that the effective average action be considered in general a function of two variables. We plan to consider these more general truncations in a future paper.

In conclusion, let us comment on the use of tetrad vs. metric variables. Since fermions exists in nature, it may seem in principle inevitable that gravity has to be described by tetrads. This would complicate the theory significantly. Every diffeomorphism-invariant functional of the metric can be viewed as a diffeomorphism and local Lorentz-invariant functional of the tetrad, but in the bimetric formalism there are many functionals of two tetrads that cannot be viewed as functionals of two metrics. Therefore, as already noted in [34], the tetrad theory space is much bigger than the metric theory space.

The necessity of using tetrads should, however, not be taken as a foregone conclusion. First of all, it is possible that the fermions occurring in nature are Kähler fermions. This would completely remove the argument for the use of tetrads, even in principle. Whether this is the case or not is a difficult issue that should be answered experimentally. For the time being one might just consider the use of Kähler fermions as a computational trick. Second, even if we stick to spinor matter, by squaring the Dirac operator and using a type II cutoff one can calculate many quantum effects due to fermions without ever having to use tetrad fields. The additional complications due to the Lorentz gauge fixing and the increased sensitivity to gauge and scheme choice advise against the 
use of the tetrad formalism, as a matter of practical convenience.

\section{Acknowledgements}

R.P. thanks U. Harst, M. Reuter and F. Saueressig for discussions and hospitality at the University of Mainz during the preparation of this paper.

\section{APPENDIX I: DIRAC SPECTRAL SUMS}

To compute the sum (28) we can use the Euler- Maclaurin formula

$$
\sum_{i=0}^{n} F(i)=\int_{0}^{n} F(x) d x-B_{1} \cdot(F(n)+F(0))+\sum_{k=1}^{p} \frac{B_{2 k}}{(2 k) !}\left(F^{(2 k-1)}(n)-F^{(2 k-1)}(0)\right)+\text { remainder }
$$

where $B_{i}$ are the Bernoulli numbers. After collecting a volume contribution, the only terms we need to compute are the 0 -th and 1 -st power of $R$. Note that only the integral depends on $R$, and therefore, in dimensions $d>2$ for the terms that we are interested in it is enough to compute the integral.

Since the volume of the $d$-sphere is $V(d)=\frac{2}{d !} \Gamma\left(\frac{d}{2}+1\right)(4 \pi)^{d / 2}\left(\frac{(d-1) d}{R}\right)^{d / 2}$ we only have to isolate the terms in the integral proportional to $R^{-d / 2}$ and $R^{1-d / 2}$

$$
22^{\left[\frac{d}{2}\right]} \int_{0}^{k \sqrt{\frac{d(d-1)}{R}}-\frac{d}{2}} \mathrm{~d} n\left(\begin{array}{c}
n+d-1 \\
n
\end{array}\right)=2 \frac{2^{\left[\frac{d}{2}\right]}}{(d-1) !} \int_{0}^{k \sqrt{\frac{d(d-1)}{R}}-\frac{d}{2}} \mathrm{~d} n(n+d-1) \cdots(n+1)
$$

changing variables $n \rightarrow n^{\prime}-d / 2$

$$
2 \frac{2^{\left[\frac{d}{2}\right]}}{(d-1) !} \int_{\frac{d}{2}}^{k \sqrt{\frac{d(d-1)}{R}}} \mathrm{~d} n^{\prime}\left(n^{\prime}+\frac{d}{2}-1\right) \cdots\left(n^{\prime}-\left(\frac{d}{2}-1\right)\right)
$$

the terms we are interested in come from the integral of the two highest order power of $n^{\prime}$

$$
\left(n^{\prime}+\frac{d}{2}-1\right) \cdots\left(n^{\prime}-\left(\frac{d}{2}-1\right)\right)=n^{\prime d-1}-n^{\prime d-3} \sum_{k=1}^{\left[\frac{d-1}{2}\right]}\left(\frac{d}{2}-k\right)^{2}+\cdots
$$

we can rewrite the sum $\sum_{k=1}^{\left[\frac{d-1}{2}\right]}\left(\frac{d}{2}-k\right)^{2}=\frac{1}{24} d(d-1)(d-2)$, and perform the integral

$\operatorname{Tr}\left[\frac{\partial_{t} R_{k}}{P_{k}}\right]=2 \frac{2^{\left[\frac{d}{2}\right]}}{(d-1) !} \frac{1}{d}\left(k \sqrt{\frac{d(d-1)}{R}}\right)^{d}-2 \frac{2^{\left[\frac{d}{2}\right]}}{(d-1) !} \frac{1}{d-2}\left(k \sqrt{\frac{d(d-1)}{R}}\right)^{d-2} \frac{1}{24} d(d-1)(d-2)+\cdots$

Collecting the volume of $\mathcal{S}^{d}$ we obtain

$$
\frac{d \Gamma_{k}}{d t}=-\operatorname{Tr}\left[\frac{\partial_{t} R_{k}}{P_{k}}\right]=-\frac{1}{\Gamma\left(\frac{d}{2}+1\right)} \frac{2^{\left[\frac{d}{2}\right]}}{(4 \pi)^{\frac{d}{2}}} V(d)\left(k^{d}-\frac{d}{24} k^{d-2} R+O\left(R^{2}\right)\right)
$$




\section{APPENDIX II: TYPE IB CALCULATION}

We report here the detailed computation of the $A$ and $B$ coefficients of (48,49) for a Type Ib cutoff. The FRGE is the sum of traces over the irreducible components of the metric fluctuation defined in (41). They give:

$$
\begin{aligned}
& \frac{1}{2} \operatorname{Tr}_{(2)} \frac{\partial_{t} R_{k}+\eta R_{k}}{P_{k}+\left(\frac{d(d-3)+4}{d(d-1)}-\zeta \frac{d-2}{2 d}\right) R-(2-\zeta) \Lambda}= \\
& \frac{1}{2} \frac{1}{(4 \pi)^{d / 2}} \int d x \sqrt{g}\left[\frac{(d-2)(d+1)}{2} Q_{\frac{d}{2}}\left(\frac{\partial_{t} R_{k}+\eta R_{k}}{P_{k}-(2-\zeta) \Lambda}\right)+\frac{(d-5)(d+1)(d+2)}{12(d-1)} Q_{\frac{d}{2}-1}\left(\frac{\partial_{t} R_{k}+\eta R_{k}}{P_{k}-(2-\zeta) \Lambda}\right) R\right. \\
& \left.-\left(\frac{d(d-3)+4}{d(d-1)}-\zeta \frac{d-2}{2 d}\right) \frac{(d-2)(d+1)}{2} Q_{\frac{d}{2}}\left(\frac{\partial_{t} R_{k}+\eta R_{k}}{\left(P_{k}-(2-\zeta) \Lambda\right)^{2}}\right) R\right] \\
& \frac{1}{2} \operatorname{Tr}_{(1)}^{\prime} \frac{\partial_{t} R_{k}+\eta R_{k}}{P_{k}+\left(\frac{\alpha(d-2)-1}{d}-\zeta \alpha \frac{d-2}{2 d}\right) R-\alpha(2-\zeta) \Lambda}= \\
& \frac{1}{2} \frac{1}{(4 \pi)^{d / 2}} \int d x \sqrt{g}\left[(d-1) Q_{\frac{d}{2}}\left(\frac{\partial_{t} R_{k}+\eta R_{k}}{P_{k}-\alpha(2-\zeta) \Lambda}\right)+\frac{(d-3)(d+2)}{6 d} Q_{\frac{d}{2}-1}\left(\frac{\partial_{t} R_{k}+\eta R_{k}}{P_{k}-\alpha(2-\zeta) \Lambda}\right) R\right. \\
& \left.-\left(\frac{\alpha(d-2)-1}{d}-\zeta \alpha \frac{d-2}{2 d}\right)(d-1) Q_{\frac{d}{2}}\left(\frac{\partial_{t} R_{k}+\eta R_{k}}{\left(P_{k}-\alpha(2-\zeta) \Lambda\right)^{2}}\right) R\right] \\
& \frac{1}{2} \operatorname{Tr}^{\prime \prime}{ }_{(0)} \frac{\partial_{t} R_{k}+\eta R_{k}}{P_{k}+\frac{\alpha(d-2)(2-\zeta)-4}{2(d-1)-\alpha(d-2)} R-\frac{\alpha d(2-\zeta)}{2(d-1)-\alpha(d-2)} \Lambda}= \\
& \frac{1}{2} \frac{1}{(4 \pi)^{d / 2}} \int d x \sqrt{g}\left[Q_{\frac{d}{2}}\left(\frac{\partial_{t} R_{k}+\eta R_{k}}{P_{k}-\frac{\alpha d(2-\zeta)}{2(d-1)-\alpha(d-2)} \Lambda}\right)+\frac{1}{6} Q_{\frac{d}{2}-1}\left(\frac{\partial_{t} R_{k}+\eta R_{k}}{P_{k}-\frac{\alpha d(2-\zeta)}{2(d-1)-\alpha(d-2)} \Lambda}\right) R\right. \\
& \left.-\frac{\alpha(d-2)(2-\zeta)-4}{2(d-1)-\alpha(d-2)} Q_{\frac{d}{2}}\left(\frac{\partial_{t} R_{k}+\eta R_{k}}{\left(P_{k}-\frac{\alpha d(2-\zeta)}{2(d-1)-\alpha(d-2)} \Lambda\right)^{2}}\right) R\right] \\
& \frac{1}{2} \operatorname{Tr}_{(0)} \frac{\partial_{t} R_{k}+\eta R_{k}}{P_{k}+\frac{d-4+\zeta}{2(d-1)-\alpha(d-2)} R-\frac{2 d\left(1+\frac{\zeta}{d-2}\right)}{2(d-1)-\alpha(d-2)} \Lambda}= \\
& \frac{1}{2} \frac{1}{(4 \pi)^{d / 2}} \int d x \sqrt{g}\left[Q_{\frac{d}{2}}\left(\frac{\partial_{t} R_{k}+\eta R_{k}}{P_{k}-\frac{2 d\left(1+\frac{\zeta}{d-2}\right)}{2(d-1)-\alpha(d-2)} \Lambda}\right)+\frac{1}{6} Q_{\frac{d}{2}-1}\left(\frac{\partial_{t} R_{k}+\eta R_{k}}{P_{k}-\frac{2 d\left(1+\frac{\zeta}{d-2}\right)}{2(d-1)-\alpha(d-2)} \Lambda}\right) R\right. \\
& \left.-\frac{d-4+\zeta}{2(d-1)-\alpha(d-2)} Q_{\frac{d}{2}}\left(\frac{\partial_{t} R_{k}+\eta R_{k}}{\left(P_{k}-\frac{2 d\left(1+\frac{\zeta}{d-2}\right)}{2(d-1)-\alpha(d-2)} \Lambda\right)^{2}}\right) R\right]
\end{aligned}
$$

Here a prime or a double prime indicate that the first or the first and the second eigenvalues have to be omitted from the trace (because $\xi_{\mu}$ and $\sigma$ obey to some differential constraints, for more details see for example [5]). The contribution of the transverse and longitudinal parts of the 
diffeomorphism ghosts are

$$
\begin{gathered}
-\operatorname{Tr}_{(1)} \frac{\partial_{t} R_{k}}{P_{k}-\frac{R}{d}}=-\frac{1}{(4 \pi)^{d / 2}} \int d x \sqrt{g}\left[(d-1) Q_{\frac{d}{2}}\left(\frac{\partial_{t} R_{k}}{P_{k}}\right)-\frac{d-1}{d} Q_{\frac{d}{2}}\left(\frac{\partial_{t} R_{k}}{P_{k}^{2}}\right) R\right. \\
\left.+\frac{(d+2)(d-3)}{6 d} Q_{\frac{d}{2}-1}\left(\frac{\partial_{t} R_{k}}{P_{k}}\right) R\right] ; \\
-\operatorname{Tr}_{(0)} \frac{\partial_{t} R_{k}}{P_{k}-\frac{2 R}{d}}=-\frac{1}{(4 \pi)^{d / 2}} \int d x \sqrt{g}\left[Q_{\frac{d}{2}}\left(\frac{\partial_{t} R_{k}}{P_{k}}\right)-\frac{2}{d} Q_{\frac{d}{2}}\left(\frac{\partial_{t} R_{k}}{P_{k}^{2}}\right) R+\frac{1}{6} Q_{\frac{d}{2}-1}\left(\frac{\partial_{t} R_{k}}{P_{k}}\right) R\right] .
\end{gathered}
$$

Collecting the coefficients of $\int \sqrt{g}$ and $-\int \sqrt{g} R$ we extract the $A$ and $B$ coefficients:

$$
\begin{aligned}
& A_{1}=\frac{1}{2} \frac{16 \pi}{(4 \pi)^{d / 2}}\left[\frac{(d-2)(d+1)}{2} \tilde{Q}_{\frac{d}{2}}\left(\frac{\partial_{t} R_{k}}{P_{k}-(2-\zeta) \Lambda}\right)+(d-1) \tilde{Q}_{\frac{d}{2}}\left(\frac{\partial_{t} R_{k}}{P_{k}-\alpha(2-\zeta) \Lambda}\right)\right. \\
& \left.+\tilde{Q}_{\frac{d}{2}}\left(\frac{\partial_{t} R_{k}}{P_{k}-\frac{\alpha d(2-\zeta)}{2(d-1)-\alpha(d-2)} \Lambda}\right)+\tilde{Q}_{\frac{d}{2}}\left(\frac{\partial_{t} R_{k}}{P_{k}-\frac{2 d\left(1+\frac{\zeta}{d-2}\right)}{2(d-1)-\alpha(d-2)} \Lambda}\right)-2 d \tilde{Q}_{\frac{d}{2}}\left(\frac{\partial_{t} R_{k}}{P_{k}}\right)\right] \\
& A_{2}=\frac{1}{2} \frac{16 \pi}{(4 \pi)^{d / 2}}\left[\frac{(d-2)(d+1)}{2} \tilde{Q}_{\frac{d}{2}}\left(\frac{R_{k}}{P_{k}-(2-\zeta) \Lambda}\right)+(d-1) \tilde{Q}_{\frac{d}{2}}\left(\frac{R_{k}}{P_{k}-\alpha(2-\zeta) \Lambda}\right)\right. \\
& \left.+\tilde{Q}_{\frac{d}{2}}\left(\frac{R_{k}}{P_{k}-\frac{\alpha d(2-\zeta)}{2(d-1)-\alpha(d-2)} \Lambda}\right)+\tilde{Q}_{\frac{d}{2}}\left(\frac{R_{k}}{P_{k}-\frac{2 d\left(1+\frac{\zeta}{d-2}\right)}{2(d-1)-\alpha(d-2)} \Lambda}\right)\right] \\
& B_{1}=\frac{1}{2} \frac{16 \pi}{(4 \pi)^{d / 2}}\left[\frac{(d-5)(d+1)(d+2)}{12(d-1)} \tilde{Q}_{\frac{d}{2}-1}\left(\frac{\partial_{t} R_{k}}{P_{k}-(2-\zeta) \Lambda}\right)\right. \\
& -\left(\frac{d(d-3)+4}{d(d-1)}-\zeta \frac{d-2}{2 d}\right) \frac{(d-2)(d+1)}{2} \tilde{Q}_{\frac{d}{2}}\left(\frac{\partial_{t} R_{k}}{\left(P_{k}-(2-\zeta) \Lambda\right)^{2}}\right) \\
& +\frac{(d-3)(d+2)}{6 d} \tilde{Q}_{\frac{d}{2}-1}\left(\frac{\partial_{t} R_{k}}{P_{k}-\alpha(2-\zeta) \Lambda}\right) \\
& -\left(\frac{\alpha(d-2)-1}{d}-\zeta \alpha \frac{d-2}{2 d}\right)(d-1) \tilde{Q}_{\frac{d}{2}}\left(\frac{\partial_{t} R_{k}}{\left(P_{k}-\alpha(2-\zeta) \Lambda\right)^{2}}\right) \\
& +\frac{1}{6} \tilde{Q}_{\frac{d}{2}-1}\left(\frac{\partial_{t} R_{k}}{P_{k}-\frac{\alpha d(2-\zeta)}{2(d-1)-\alpha(d-2)} \Lambda}\right)-\frac{\alpha(d-2)(2-\zeta)-4}{2(d-1)-\alpha(d-2)} \tilde{Q}_{\frac{d}{2}}\left(\frac{\partial_{t} R_{k}}{\left(P_{k}-\frac{\alpha d(2-\zeta)}{2(d-1)-\alpha(d-2)} \Lambda\right)^{2}}\right) \\
& +\frac{1}{6} \tilde{Q}_{\frac{d}{2}-1}\left(\frac{\partial_{t} R_{k}}{P_{k}-\frac{2 d\left(1+\frac{\zeta}{d-2}\right)}{2(d-1)-\alpha(d-2)} \Lambda}\right)-\frac{d-4+\zeta}{2(d-1)-\alpha(d-2)} \tilde{Q}_{\frac{d}{2}}\left(\frac{\partial_{t} R_{k}}{\left(P_{k}-\frac{2 d\left(1+\frac{\zeta}{d-2}\right)}{2(d-1)-\alpha(d-2)} \Lambda\right)^{2}}\right) \\
& \left.-\frac{d^{2}-6}{3 d} \tilde{Q}_{\frac{d}{2}-1}\left(\frac{\partial_{t} R_{k}}{P_{k}}\right)-2 \frac{d+1}{d} \tilde{Q}_{\frac{d}{2}}\left(\frac{\partial_{t} R_{k}}{P_{k}^{2}}\right)\right]
\end{aligned}
$$




$$
\begin{aligned}
& B_{2}=\frac{1}{2} \frac{16 \pi}{(4 \pi)^{d / 2}}\left[\frac{(d-5)(d+1)(d+2)}{12(d-1)} \tilde{Q}_{\frac{d}{2}-1}\left(\frac{R_{k}}{P_{k}-(2-\zeta) \Lambda}\right)\right. \\
& \quad-\left(\frac{d(d-3)+4}{d(d-1)}-\zeta \frac{d-2}{2 d}\right) \frac{(d-2)(d+1)}{2} \tilde{Q}_{\frac{d}{2}}\left(\frac{R_{k}}{\left(P_{k}-(2-\zeta) \Lambda\right)^{2}}\right) \\
&+\frac{(d-3)(d+2)}{6 d} \tilde{Q}_{\frac{d}{2}-1}\left(\frac{R_{k}}{P_{k}-\alpha(2-\zeta) \Lambda}\right) \\
&-\left(\frac{\alpha(d-2)-1}{d}-\zeta \alpha \frac{d-2}{2 d}\right)(d-1) \tilde{Q}_{\frac{d}{2}}\left(\frac{R_{k}}{\left(P_{k}-\alpha(2-\zeta) \Lambda\right)^{2}}\right) \\
&+\frac{1}{6} \tilde{Q}_{\frac{d}{2}-1}\left(\frac{R_{k}}{P_{k}-\frac{\alpha d(2-\zeta)}{2(d-1)-\alpha(d-2)} \Lambda}\right)-\frac{\alpha(d-2)(2-\zeta)-4}{2(d-1)-\alpha(d-2)} \tilde{Q}_{\frac{d}{2}}\left(\frac{R_{k}}{\left(P_{k}-\frac{\alpha d(2-\zeta)}{2(d-1)-\alpha(d-2)} \Lambda\right)^{2}}\right) \\
&\left.+\frac{1}{6} \tilde{Q}_{\frac{d}{2}-1}\left(\frac{R_{k}}{P_{k}-\frac{2 d\left(1+\frac{\zeta}{d-2}\right)}{2(d-1)-\alpha(d-2)} \Lambda}\right)-\frac{R_{k}}{2(d-1)-\alpha(d-2)} \tilde{Q}_{\frac{d}{2}}\left(\frac{2 d\left(1+\frac{\zeta}{d-2}\right)}{\left(P_{k}-\frac{1(d-1)}{2(d-1)-\alpha(d-2)}\right)^{2}}\right)\right]
\end{aligned}
$$

Here we have defined the dimensionless versions of the $Q$-functionals: $\tilde{Q}_{\frac{d}{2}}=k^{-d} Q_{\frac{d}{2}}$ and $\tilde{Q}_{\frac{d}{2}-1}=$ $k^{2-d} Q_{\frac{d}{2}-1}$.

Finally let us consider the contribution of Lorentz ghosts. They do not propagate and therefore are usually neglected in the evaluation of the effective action in perturbation theory. Nevertheless if, following [34], we impose a cutoff on their determinant they contribute to the r.h.s. of the FRGE an amount

$$
-\operatorname{Tr} \frac{\partial_{t} R_{k}}{R_{k}+\frac{2 \mu^{2}}{\sqrt{\zeta}}}=-\frac{1}{(4 \pi)^{d / 2}} \frac{d(d-1)}{2} \int d x \sqrt{g}\left[Q_{\frac{d}{2}}\left(\frac{\partial_{t} R_{k}}{R_{k}+\frac{2 \mu^{2}}{\sqrt{\zeta}}}\right)+\frac{1}{6} Q_{\frac{d}{2}-1}\left(\frac{\partial_{t} R_{k}}{R_{k}+\frac{2 \mu^{2}}{\sqrt{\zeta}}}\right) R\right]
$$

Here we have introduced the arbitrary mass parameter $\mu$ (denoted $\bar{\mu}$ in [34]). In particular note that in the limit $\mu \rightarrow \infty$ the ghost contribution vanishes and one recovers the standard perturbative result where the Lorentz ghosts are neglected. Let $A^{L}$ and $B^{L}$ be the contribution of the Lorentz ghosts to the coefficients $A_{1}$ and $B_{1}$, defined in (48,49). From the above we read off

$$
A^{L}=-\frac{16 \pi}{(4 \pi)^{d / 2}} \frac{d(d-1)}{2} \tilde{Q}_{\frac{d}{2}}\left(\frac{\partial_{t} R_{k}}{R_{k}+\frac{2 \mu^{2}}{\sqrt{\zeta}}}\right) ; \quad B^{L}=-\frac{16 \pi}{(4 \pi)^{d / 2}} \frac{d(d-1)}{12} \tilde{Q}_{\frac{d}{2}-1}\left(\frac{\partial_{t} R_{k}}{R_{k}+\frac{2 \mu^{2}}{\sqrt{\zeta}}}\right) .
$$

Note the appearance of $R_{k}$ instead of $P_{k}$ in the denominators. In general the $Q$ functionals $Q_{n}\left(\frac{\partial_{t} R_{k}}{R_{k}+2 \mu^{2} / \sqrt{\zeta}}\right)$ can be computed explicitly, with cutoff (3), in terms of hypergeometric functions. 
For the calculations in four dimensions we only need the following

$$
\begin{aligned}
& \tilde{Q}_{1}\left(\frac{\partial_{t} R_{k}}{R_{k}+\frac{2 \mu^{2}}{\sqrt{\zeta}}}\right)=\log \left(1+\frac{\sqrt{\zeta}}{2 \tilde{\mu}^{2}}\right) ; \\
& \tilde{Q}_{2}\left(\frac{\partial_{t} R_{k}}{R_{k}+\frac{2 \mu^{2}}{\sqrt{\zeta}}}\right)=-1+\left(1+\frac{2 \tilde{\mu}^{2}}{\sqrt{\zeta}}\right) \log \left(1+\frac{\sqrt{\zeta}}{2 \tilde{\mu}^{2}}\right) .
\end{aligned}
$$

\section{APPENDIX III: TYPE IIB CALCULATION}

We report here the $A$ and $B$ coefficients of (48,49) for a Type IIb cutoff. The contributions of the irreducible components of the metric fluctuation to the r.h.s. of the FRGE are

$$
\begin{aligned}
& \frac{1}{2} \operatorname{Tr}_{(2)} \frac{\partial_{t} R_{k}+\eta R_{k}}{P_{k}-(2-\zeta) \Lambda}=\frac{1}{2} \frac{1}{(4 \pi)^{d / 2}} \int d x \sqrt{g}\left[\frac{(d-2)(d+1)}{2} Q_{\frac{d}{2}}\left(\frac{\partial_{t} R_{k}+\eta R_{k}}{P_{k}-(2-\zeta) \Lambda}\right)\right. \\
& \left.-\frac{(d+1)}{12 d}\left(5 d^{2}-22 d+48-3 \zeta(d-2)^{2}\right) Q_{\frac{d}{2}-1}\left(\frac{\partial_{t} R_{k}+\eta R_{k}}{P_{k}-(2-\zeta) \Lambda}\right) R\right] \\
& \frac{1}{2} \operatorname{Tr}_{(1)}^{\prime} \frac{\partial_{t} R_{k}+\eta R_{k}}{P_{k}-\alpha(2-\zeta) \Lambda}=\frac{1}{2} \frac{1}{(4 \pi)^{d / 2}} \int d x \sqrt{g}\left[(d-1) Q_{\frac{d}{2}}\left(\frac{\partial_{t} R_{k}+\eta R_{k}}{P_{k}-\alpha(2-\zeta) \Lambda}\right)\right. \\
& \left.-\frac{\left(d^{2}+5 d-12+3 \alpha(\zeta-2)(d-1)(d-2)\right)}{6 d} Q_{\frac{d}{2}-1}\left(\frac{\partial_{t} R_{k}+\eta R_{k}}{P_{k}-\alpha(2-\zeta) \Lambda}\right) R\right] \\
& \frac{1}{2} \operatorname{Tr}^{\prime \prime}{ }_{(0)} \frac{\partial_{t} R_{k}+\eta R_{k}}{P_{k}-\frac{\alpha d(2-\zeta)}{2(d-1)-\alpha(d-2)} \Lambda}=\frac{1}{2} \frac{1}{(4 \pi)^{d / 2}} \int d x \sqrt{g}\left[Q_{\frac{d}{2}}\left(\frac{\partial_{t} R_{k}+\eta R_{k}}{P_{k}-\frac{\alpha d(2-\zeta)}{2(d-1)-\alpha(d-2)} \Lambda}\right)\right. \\
& \left.+\frac{14+(2-7 \alpha-3 \zeta \alpha)(d-2)}{6(2(d-1)-\alpha(d-2))} Q_{\frac{d}{2}-1}\left(\frac{\partial_{t} R_{k}+\eta R_{k}}{P_{k}-\frac{\alpha d(2-\zeta)}{2(d-1)-\alpha(d-2)} \Lambda}\right) R\right] \\
& \frac{1}{2} \operatorname{Tr}_{(0)} \frac{\partial_{t} R_{k}+\eta R_{k}}{P_{k}-\frac{2 d\left(1+\frac{\zeta}{d-2}\right)}{2(d-1)-\alpha(d-2)} \Lambda}=\frac{1}{2} \frac{1}{(4 \pi)^{d / 2}} \int d x \sqrt{g}\left[Q_{\frac{d}{2}}\left(\frac{\partial_{t} R_{k}+\eta R_{k}}{P_{k}-\frac{2 d\left(1+\frac{\zeta}{d-2}\right)}{2(d-1)-\alpha(d-2)} \Lambda}\right)\right. \\
& \left.+\frac{22-4 d+(2-d) \alpha-6 \zeta}{6(2(d-1)-\alpha(d-2))} Q_{\frac{d}{2}-1}\left(\frac{\partial_{t} R_{k}+\eta R_{k}}{P_{k}-\frac{2 d\left(1+\frac{\zeta}{d-2}\right)}{2(d-1)-\alpha(d-2)} \Lambda}\right) R\right]
\end{aligned}
$$

The contribution of the diffeomorphism ghosts is

$$
\begin{aligned}
& -\operatorname{Tr}_{(1)} \frac{\partial_{t} R_{k}}{P_{k}}=-\frac{1}{(4 \pi)^{d / 2}} \int d x \sqrt{g}\left[(d-1) Q_{\frac{d}{2}}\left(\frac{\partial_{t} R_{k}}{P_{k}}\right)+\frac{d^{2}+5 d-12}{6 d} R Q_{\frac{d}{2}-1}\left(\frac{\partial_{t} R_{k}}{P_{k}}\right)\right] \\
& -\operatorname{Tr}_{(0)} \frac{\partial_{t} R_{k}}{P_{k}}=-\frac{1}{(4 \pi)^{d / 2}} \int d x \sqrt{g}\left[Q_{\frac{d}{2}}\left(\frac{\partial_{t} R_{k}}{P_{k}}\right)+\frac{d+12}{6 d} R Q_{\frac{d}{2}-1}\left(\frac{\partial_{t} R_{k}}{P_{k}}\right)\right] .
\end{aligned}
$$


The contribution of Lorentz ghosts is the same as in the Type Ib case. The coefficients $A_{1}$ and $A_{2}$ are the same as in equations (78, (79), whereas

$$
\begin{aligned}
B_{1}=\frac{1}{2} \frac{16 \pi}{(4 \pi)^{d / 2}[} & -\frac{(d+1)}{12 d}\left(5 d^{2}-22 d+48-3 \zeta(d-2)^{2}\right) \tilde{Q}_{\frac{d}{2}-1}\left(\frac{\partial_{t} R_{k}}{P_{k}-(2-\zeta) \Lambda}\right) \\
& +\frac{d^{2}+5 d-12+3 \alpha(\zeta-2)(d-1)(d-2)}{6 d} \tilde{Q}_{\frac{d}{2}-1}\left(\frac{\partial_{t} R_{k}}{P_{k}-\alpha(2-\zeta) \Lambda}\right) \\
& +\frac{22-4 d+(2-d) \alpha-6 \zeta}{6(2(d-1)-\alpha(d-2))} \tilde{Q}_{\frac{d}{2}-1}\left(\frac{\partial_{t} R_{k}}{P_{k}-\frac{2 d\left(1+\frac{\zeta}{d-2}\right)}{2(d-1)-\alpha(d-2)} \Lambda}\right) \\
& \left.+\frac{14+(2-7 \alpha-3 \zeta \alpha)(d-2)}{6(2(d-1)-\alpha(d-2))} \tilde{Q}_{\frac{d}{2}-1}\left(\frac{\partial_{t} R_{k}}{P_{k}-\frac{\alpha d(2-\zeta)}{2(d-1)-\alpha(d-2)} \Lambda}\right)-\frac{d+6}{3} \tilde{Q}_{\frac{d}{2}-1}\left(\frac{\partial_{t} R_{k}}{P_{k}}\right)\right] \\
B_{2}=\frac{1}{2} \frac{16 \pi}{(4 \pi)^{d / 2}[} & -\frac{(d+1)}{12 d}\left(5 d^{2}-22 d+48-3 \zeta(d-2)^{2}\right) \tilde{Q}_{\frac{d}{2}-1}\left(\frac{R_{k}}{P_{k}-(2-\zeta) \Lambda}\right) \\
& +\frac{d^{2}+5 d-12+3 \alpha(\zeta-2)(d-1)(d-2)}{6 d} \tilde{Q}_{\frac{d}{2}-1}\left(\frac{R_{k}}{P_{k}-\alpha(2-\zeta) \Lambda}\right) \\
& +\frac{22-4 d+(2-d) \alpha-6 \zeta}{6(2(d-1)-\alpha(d-2))} \tilde{Q}_{\frac{d}{2}-1}\left(\frac{R_{k}}{P_{k}-\frac{2 d\left(1+\frac{\zeta}{d-2}\right)}{2(d-1)-\alpha(d-2)} \Lambda}\right) \\
& \left.+\frac{14+(2-7 \alpha-3 \zeta \alpha)(d-2)}{6(2(d-1)-\alpha(d-2))} \tilde{Q}_{\frac{d}{2}-1}\left(\frac{R_{k}}{P_{k}-\frac{\alpha d(2-\zeta)}{2(d-1)-\alpha(d-2)} \Lambda}\right)\right]
\end{aligned}
$$

[1] F.J. Wegner, A. Houghton, Phys. Rev. A8 401-412 (1973).

J. Polchinski, Nucl. Phys. B231 269-295 (1984).

T. R. Morris, Phys. Lett. B 329 (1994) 241 arXiv:hep-ph/9403340.

[2] C. Wetterich, Phys. Lett. B 301 (1993) 90.

[3] M. Reuter, Phys. Rev. D 57 (1998) 971, arXiv:hep-th/9605030.

[4] D. Dou and R. Percacci, Class. Quant. Grav. 15 (1998) 3449, arXiv:hep-th/9707239.

[5] A. Codello, R. Percacci, and C. Rahmede, Ann. Phys. 324 (2009) 44, arXiv:0805.2909(hep-th)].

[6] W. Souma, Prog. Theor. Phys. 102 (1999) 181, arXiv:hep-th/9907027.

[7] O. Lauscher and M. Reuter, Phys. Rev. D 65 (2002) 025013, arXiv:hep-th/0108040;

[8] M. Reuter and F. Saueressig, Phys. Rev. D 65 (2002) 065016, arXiv:hep-th/0110054.

[9] D.F. Litim, Phys. Rev. D 64 (2001) 105007, arXiv:hep-th/0103195; Phys. Rev. Lett. 92 (2004) 201301, arXiv:hep-th/0312114. P. Fischer and D.F. Litim, Phys. Lett. B 638 (2006) 497, arXiv:hep-th/0602203.

[10] D. Benedetti, New J. Phys. 14, 015005 (2012) arXiv:1107.3110 [hep-th]

[11] A. Eichhorn, H. Gies, and M. Scherer, Phys. Rev. D 80 (2009) 104003, arXiv:0907.1828(hep-th)].

[12] K. Groh and F. Saueressig(2010) J. Phys. A43 365403 (2010). arXiv:1001.5032 [hep-th]

[13] A. Eichhorn, H. Gies (2010) Phys. Rev. D81, 104010 (2010) arXiv:1001.5033 [hep-th]

[14] I. Donkin and J. Pawlowski, arXiv:1203.4207 [hep-th] 
[15] O. Lauscher and M. Reuter, Phys. Rev. D 66 (2002) 025026, arXiv:hep-th/0205062;

[16] A. Codello and R. Percacci, Phys. Rev. Lett. 97 (2006) 221301, arXiv:hep-th/0607128.

[17] M.R. Niedermaier, Phys. Rev. Lett. 103 (2009) 101303; Nucl. Phys. B 833 (2010) 226-270.

[18] A. Codello, R. Percacci, and C. Rahmede, Int. J. Mod. Phys. A 23 (2008) 143, arXiv:0705.1769 (hep-th)].

[19] P.F. Machado and F. Saueressig, Phys. Rev. D 77 (2008) 124045, arXiv:0712.0445(hep-th)].

[20] D. Benedetti, P.F. Machado, and F. Saueressig, Mod. Phys. Lett. A 24 (2009) 2233, arXiv:0901.2984(hep-th)]

[21] R. Percacci and D. Perini, Phys. Rev. D 67 (2003) 081503, arXiv:hep-th/0207033.

[22] R. Percacci and D. Perini, Phys. Rev. D 68 (2003) 044018, arXiv:hep-th/0304222.

[23] G. Narain and R. Percacci, Class. Quant. Grav. 27 (2010) 075001, arXiv:0911.0386(hep-th)]; G. Narain and C. Rahmede, Class. Quant. Grav. 27 (2010) 075002, arXiv:0911.0394(hep-th)].

[24] D. Benedetti, P.F. Machado, and F. Saueressig, Nucl. Phys. B 824 (2010) 168, arXiv:0902.4630(hep-th)].

[25] R. Percacci, Phys. Rev. D 73 (2006) 041501, arXiv:hep-th/0511177.

[26] O. Zanusso, L. Zambelli, G.P. Vacca and R. Percacci, Phys. Lett. B689 90-94 (2010) arXiv:0904.0938 [hep-th]

[27] A. Rodigast and T. Schuster, Phys. Rev. Lett. 104, 081301 (2010) arXiv:0908.2422 [hep-th]

[28] O. Zanusso, G.P. Vacca, Phys. Rev. Lett. 105, 231601 (2010) arXiv:1009.1735 [hep-th]

[29] A. Eichhorn and H. Gies, New J. Phys. 13 (2011) 125012 [arXiv:1104.5366 [hep-th]].

[30] G. Narain and R. Percacci, Acta Phys. Polon. B40 (2009) 3439-3457 To appear in the proceedings of Conference: C09-05-31.2 e-Print: arXiv:0910.5390 [hep-th]

[31] F. Larsen, F. Wilczek, Nucl. Phys. B 458 249-266 (1996) arXiv:hep-th/9506066 [hep-th]

[32] X. Calmet, D. Reeb and S. Hsu Phys. Rev. Lett. 101171802 (2008) arXiv:0805.0145 [hep-ph]

[33] E. Manrique, M. Reuter and A. Saueressig, Ann. of Phys. 325 785-815 (2010) arXiv:0907.2617 [hep-th] ibid. 326 440-462 (2011) arXiv:1003.5129 [hep-th] ibid. 326 463-485 (2011) arXiv:1006.0099 [hep-th]

[34] U. Harst and M. Reuter, arXiv:1203.2158 [hep-th]].

[35] E. Kähler, Rendiconti di Matematica (3-4) 21 (1962) 425.

[36] W. Graf, Annales Inst. Poincare Phys.Theor. 29 85-109 (1978)

[37] I.M. Benn and R.W. Tucker Comm. math. Phys. 89341 (1983)

[38] C. Rahmede, PhD thesis, SISSA (2008).

[39] D. Litim, A. Satz arXiv:1205.4218 [hep-th] 